\title{
A REVIEW OF THE STATISTICAL THEORY OF TURBULENCE*
}

\author{
BY \\ HUGH L. DRYDEN \\ National Bureau of Standards
}

1. Introduction. The irregular random motion of small fluid masses to which the name turbulence is given is of such complexity that there can be no hope of a theory which will describe in detail the velocity and pressure fields at every instant. Existing theories may be classified as either empirical or statistical.

In the empirical theories attention is focused only on the distribution of mean speed and mean pressure, and assumptions are made as to the dependence of the shearing stresses required to satisfy the equations of motion of the mean flow. These assumptions involve one or more empirical constants. While the type of assumption adopted is of ten selected on the basis of some hypothesis as to the character of the fluctuations of speed and pressure, the theory rests on the final assumption rather than on the hypothesis as to the fluctuations. The various "mixing length" theories are of this type.

In the statistical theories consideration is given to the frequency distribution and mean values of the pressure and of the components of the velocity fluctuations, i.e. to the statistical properties of the fluctuations, and to the relation between the mean motion and these statistical properties.

Some attempts have been made to apply the methods of statistical mechanics of discrete particles. In all such attempts it is necessary to select certain discrete elements corresponding to the particles, and to make some assumption as to the probability of occurrence of various values of associated properties or more directly the frequency distribution of the associated properties. Difficulties are encountered at both points. The best known theory of this type is that of Burgers ${ }^{1}$ who selected as elements in two-dimensional flow the points in a square network of equally spaced points and as associated property the value of the stream function. This theory has not as yet led to useful results and is not satisfactory to Burgers himself. Other attempts of

* Received Nov. 19, 1942.

1 Burgers, J. M., On the application of statistical mechanics to the theory of turbulent fluid motion, I to VII, inclusive, Verh. Kon. Akad. v. Wetensch. Amsterdam 32, 414, 643, 818 (1929); 36, 276, 390, 487, 620 (1933). Summarized by Trubridge in Reports Phys. Soc. London, 1934, p. 43. 
this nature have been made by von Kármán, ${ }^{2}$ Noether, ${ }^{3}$ Tollmien, ${ }^{4}$ Gebelein, ${ }^{5}$ Dedebant, Wehrlé and Schereschewsky, ${ }^{6}$ and Takahasi. ${ }^{7}$

Many of the statistical theories just mentioned do not require the turbulent fluctuations to satisfy the equations of motion nor do they require the fluid motion to be continuous. A statistical theory of turbulence which is applicable to continuous movements and which satisfies the equations of motion was inaugurated in 1935 by Taylor ${ }^{8}$ and further developed by himself and by von Kármán. ${ }^{9}$ It is the object of this paper to give a connected account of the present state of this particular statistical theory of turbulence.

2. Turbulent fluctuations and the mean motion. As in other theories of turbulent flow, the flow is regarded as a mean motion with velocity components, $U, V$, and $W$, on which are superposed fluctuations of the velocity with components of magnitude $u, v$, and $w$ at any instant. The mean values of $u, v$, and $w$ are zero. In most cases $U, V$, and $W$ are the average values at a fixed point over a definite period of time, although in certain problems it is more convenient to take averages over a selected area or within a selected volume at a given instant. The rules for forming mean values were stated by Reynolds $^{10}$ and some further critical discussion by Burgers and others has been recorded in connection with a lecture by Oseen. ${ }^{11}$

When the turbulent motion is produced in a pipe by the action of a constant pressure gradient or near the surface of an object in a wind tunnel in which the fan is operated at a constant speed, there is considerable freedom

${ }^{2}$ Kármán, Th. von, Über die Stabilität der Laminarströmung und die Theorie der Turbulenz, Proc. 1st Inter. Congr. Appl. Mech., Delft, 1924, p. 97.

${ }^{3}$ Noether, F., Dynamische Gesichtspunkte zu einer statistischen Turbulenztheorie, Z. angew. Math. u. Mech. 13, 115 (1933).

4 Tollmien, W., Der Burgersche Phasenraum und einige Fragen der Turbulenzstatistik, Z. angew. Math. u. Mech. 13, 331 (1933). Brief abstract of this paper entitled, On the turbulence statistics in Burgers' phase space, Physics, 4, 289 (1933).

${ }^{5}$ Gebelein, H., Turbulenz: Physikalische Statistik und Hydrodynamik, Julius Springer, Berlin, 1935.

${ }^{6}$ Dedebant, G., Wehrle, $\mathrm{Ph} .$, and Schereschewsky, $\mathrm{Ph} .$, Le maximum de probabilité dans les mouvements permanents. Application d la turbulence, Comptes Rendus Ac. Sci. Paris 200, 203 (1935). Also Dedebant, G., and Wehrlé, Ph., Sur les équations aux valeurs probables d'un fluide turbulent, Comptes Rendus Ac. Sci. Paris 206, 1790 (1938).

7 Takahasi, K., On the theory of turbulence, The Geophysical Magazine 10, 1 (1936).

8 Taylor, G. I., Statistical theory of turbulence, I-V inclusive, Proc. Roy. Soc. London Ser. A, 151, 421 (1935) and 156, 307 (1936). Also, The statistical theory of isotropic turbulence, Jour. Aeron. Sci., 4, 311 (1937).

' Karmán, Th. von, On the statistical theory of turbulence, Proc. Nat. Acad. Sci. 23, 98 (1937). Also The fundamentals of the statistical theory of turbulence, Jour. Aeron. Sci. 4, 131 (1937). Also with Howarth, L., On the statistical theory of isotropic turbulence, Proc. Roy. Soc. London Ser. A, 164, 192 (1938).

${ }^{10}$ Reynolds, O., On the dynamical theory of incompressible viscous fluids and the determination of the criterion, Phil. Trans. Roy. Soc. London 186, 123 (1895).

"Oseen, C. W., Das Turbulenzproblem, Proc. 3rd Inter. Congr. Appl. Mech., Stockholm, 1931, vol. 1, p. 3. 
in selecting the time interval for which mean values are taken. So long as the time interval is longer than some fixed value dependent on the scale of the apparatus and the speed, the mean values are independent of the magnitude of the time interval selected and there is a clear separation between the turbulent fluctuations and the mean motion. If the mean motion itself is "slowly" variable, as in the case of the natural wind, difficulty arises; the separation becomes imperfect and arbitrary. The slowly variable mean may be taken over time intervals of five minutes, one day, or ten years according to the object of the study and the magnitude of the turbulent fluctuations varies accordingly. Even in flows under constant pressure gradient, there will usually be some experimental difficulty in maintaining the conditions absolutely constant, and the question will naturally arise as to how the fluctuations arising from this source may be eliminated from the "true" turbulent fluctuations.

3. Vortex trails. For a long time every flow in which "fast" fluctuations of velocity occurred was regarded as a turbulent flow but experimental measurements of fluctuations show several identifiable types. The experimental results suggest the limitation of the term "turbulent fluctuation" to one of these types characterized by the random nature of the fluctuations. This random characteristic is in marked contrast with the regularity and periodicity noted in a second type of fluctuation associated with vortex trails.

It is well known that when a cylinder or other object of blunt cross section is exposed to a fluid stream, a vortex trail appears under certain circumstances, vortices breaking away with a regular periodicity. The speed fluctuations observed in the trail are periodic and in themselves do not produce turbulent mixing. At comparatively short distances the regular pattern transforms into an irregular turbulent motion, but the fluctuations within the trail itself do not have the character of the final turbulent fluctuations.

The fluctuations of turbulence are irregular, without definite periodicity with time. The amplitude distribution corresponds to the Gaussian distribution, i.e. the number of times during a long time interval that a given magnitude of fluctuation is reached varies with the magnitude according to the "error" curve.

If this randomness is regarded as an essential feature of the turbulent fluctuations, turbulence is not equivalent to any regular vortex system however complex. The equivalent vortex picture is a large family of vortex systems, whose statistical properties only, not individual histories, are significant.

4. Space and time averages. The speed fluctuations $u, v$, and $w$, though designated the fluctuations at a point, are in reality averages throughout a certain volume and over a certain time as are the speed components in the usual hydrodynamic theory. The volume is small in comparison with the dimensions of interest in the flow but large enough to include many molecules. A cube of size $0.001 \mathrm{~mm}$, containing at atmospheric pressure about $2.7 \times 10^{7}$ molecules, satisfies this condition. The time interval is short in com- 
parison with any time interval of interest in the mean properties of the flow but long in comparison with the time required for a molecule to traverse the mean free path. The number of collisions at atmospheric pressure is of the order of $5 \times 10^{9}$ per second and hence a time interval of $10^{-6}$ seconds would suffice.

No instruments have yet been constructed to give values averaged over so small a volume or so short a time interval. The best performance obtained to date is that of hot wire anemometers which have been developed to the point where average values over a cylindrical volume perhaps $0.01 \mathrm{~mm}$ in diameter and $1 \mathrm{~mm}$ long and over a time interval of approximately $0.5 \times 10^{-3}$ seconds can be obtained. Experimental results show that averages over these space and time intervals are not appreciably different from those for somewhat larger space and time intervals and suggest that averages over smaller intervals would not be appreciably different. The results also suggest that measuring equipment that does not approach these space and time intervals gives results which largely reflect the properties of the measuring instrument rather than the properties of the turbulent fluctuations. In other words the measurement is that of a variable mean velocity over space and time intervals fixed by the characteristics of the instrument, rather than measurements of the turbulent fluctuations. If the frequency spectrum of the turbulent fluctuations is known, the effect of the instrument characteristics can be estimated, as discussed in section 19.

5. Pulsations. Reference has previously been made to the difficulty in certain cases of making a clear separation between the mean motion and the turbulent fluctuations, because of the difficulty of defining a time interval long enough to include many fluctuations but small enough so that the mean varies only slowly. The difficulty is often increased by the presence of a fairly rapid variation of the mean speed over large areas, perhaps the entire cross section of the fluid stream, to which the name pulsation may be given. Such a fluctuation is recognizable by the fact that there is a regularity in the space distribution of the fluctuations such that definite phase relations exist. Pulsations have been observed in laminar flow in boundary layers. An essential characteristic of the turbulent fluctuations is an irregularity and randomness in the space distribution as well as in the time distribution.

It is of ten possible to eliminate the effect of pulsations on the measurements by a low frequency cut-off in the equipment for measuring $u, v$, and $w$. The choice of the cut-off frequency is equivalent to a selection of the time interval over which averages are taken to obtain the mean speed and by this device the pulsations are regarded as variations of the mean speed.

6. Continuity of the turbulent motion. It is well known that the structure of a fluid is in the final analysis discontinuous, the fluid consisting of individual molecules. Nevertheless the usual hydrodynamic theory regards the fluid 
as a continuum. Such an assumption can be justified when the dimensions of the flow system are very large compared to the mean free path of the molecules. The velocity of the fluid at any point is then defined as the vector average of the velocities of the molecules in a small volume surrounding the point, the value obtained being independent of the magnitude and shape of the volume within certain limits.

Some investigators ${ }^{12}$ have concluded that the phenomena of turbulence require the assumption of discontinuity in the instantaneous components. The Taylor-von Kármán statistical theory retains the assumption that the fluctuations are continuous functions of space and time as in Reynolds' theory.

The applicability of this assumption is a matter for experimental determination. If experimentally a volume and time interval can be selected which may be regarded as large in comparison with molecular distances and periods but small as compared to the volumes and time intervals of interest in the turbulent fluctuations, the fluctuations may be safely regarded as continuous. As described in section 4, the experimental data perhaps do not prove but do definitely suggest that such a choice is possible and to that extent the assumption of continuity is experimentally justified.

7. The Reynolds stresses. If in the Navier-Stokes equations of motion the components of the velocity are written as $U+u, V+v, W+w$, thus regarding the motion as a mean motion $U, V, W$, with fluctuations $u, v, w$ superposed, and mean values taken in accordance with the rules mentioned in section 2 , a new set of equations is obtained which differs from the first only in the presence of additional terms added to the mean values of the stresses due to viscosity. These additional terms are called the Reynolds stresses or eddy stresses. The eddy normal stress components are $-\rho \overline{u^{2}},-\rho \overline{v^{2}},-\rho \overline{w^{2}}$ and the eddy shearing stress components are $-\rho \overline{u v},-\rho \overline{v w},-\rho \overline{u w}$. Each stress component is thus equal to the rate of transfer of momentum across the corresponding surface by the fluctuations.

In the light of kinetic theory the eddy stresses closely parallel in origin the viscous stresses. It has been explained how $u, v$, and $w$ are themselves the mean speeds of many molecules. The effect of the molecular motions appears in the smoothed equations of the continuum as a stress, the components of which are equal to the rate of transfer of momentum by the molecules across the corresponding surfaces.

8. Correlation. If the fluctuations were perfectly random, the eddy shearing stress components $-\rho \overline{u v},-\rho \overline{v w},-\rho \overline{u w}$ would be zero. The existence of eddy shearing stresses is dependent on the existence of a correlation between the several components of the velocity fluctuation at any given point. The coefficient of correlation between $u$ and $v$ is defined as

12 Kampé de Fériet, J., Some recent researches on turbulence, Proc. Fifth Inter. Congr. Appl. Mech., Cambridge, Mass.,1938, p. 352. 


$$
R_{u v}=\frac{\overline{u v}}{\sqrt{\overline{u^{2}}} \sqrt{\overline{v^{2}}}} \cdot
$$

The mean values $\sqrt{\overline{u^{2}}}, \sqrt{\overline{v^{2}}}$, and $\sqrt{\overrightarrow{w^{2}}}$ are often called the components of the intensity of the fluctuations.

The eddy shearing stress may be written in terms of the correlation coefficient as

$$
-\rho \overline{u v}=-\rho R_{u v} \sqrt{u^{2}} \sqrt{v^{2}}
$$

and similarly for the other components.

In addition to the correlation between the components of the velocity fluctuations at a given point, the Taylor-von Kármán theory makes much use of correlations between the components of the velocity fluctuations at neighboring points. Denote the components of the fluctuations at one point by $u_{1}, v_{1}, w_{1}$, and at another point by $u_{2}, v_{2}, w_{2}$. The coefficient of correlation between $u_{1}$ and $v_{2}$ is defined as

$$
R_{u_{1} v_{2}}=\frac{\overline{u_{1} v_{2}}}{\sqrt{\overline{u_{1}^{2}} \sqrt{v_{2}^{2}}}}
$$

and similarly for any other pair. These correlation coefficients form useful tools to describe the statistical properties of the fluctuations with respect to their spatial distribution and phase relationships.

9. Scale of turbulence. The earliest attempt to describe the spatial characteristics of turbulence was the introduction of the mixing length concept, the mixing length being analogous to the mean free path of the kinetic theory of gases. Logical difficulties arise because there are no discrete fluid particles in the turbulent flow which retain their identity. A method of avoiding these difficulties was suggested by Taylor ${ }^{13}$ many years ago. He showed that the diffusion of particles starting from a point depends on the correlation $R_{t}$ between the velocity of a fluid particle at any instant and that of the same particle after a time interval $t$. If the functional relationship between $R_{t}$ and $t$ is of such a character that $R_{t}$ falls to zero at some interval $T$ and remains so for greater intervals, it is possible to define a length $l_{1}$ by the relation:

$$
l_{1}=\sqrt{v^{2}} \int_{0}^{T} R_{t} d t=\sqrt{v^{2}} \int_{0}^{\infty} R_{t} d t
$$

in which $v$ is the component of the velocity fluctuations transverse to the mean flow and in the direction in which the diffusion is studied.

${ }^{13}$ Taylor, G. I., Diffusion by continuous movements, Proc. London Math. Soc. Ser. A, 20, 196 (1921). 
This method of assigning a scale to turbulence is of value in the study of diffusion as described in section 22. It is based on the Lagrangian manner of describing the flow by following the paths of fluid particles. It is more common to use the Eulerian description by considering the stream lines existing in space at any instant. Taylor later ${ }^{8}$ suggested a method of describing the scale in the Eulerian system based on the variation of the correlation coefficient $R_{\boldsymbol{y}}$ between the values of the component $u$ at two points, separated by the distance $y$ in the direction of the $y$ coordinate, as $y$ is varied. The curve of $R_{y}$ against $y$ represents the statistical distribution of $u$ along the $y$ axis at any instant. If $R_{y}$ falls to zero and remains zero, a length $L$ may be defined by the relation:

$$
L=\int_{0}^{\infty} R_{y} d y
$$

The length $L$ is considered a possible definition of the average size of the eddies present and has been found to be a most useful measure of the scale of the turbulence, especially for the case of isotropic turbulence. Correspondingly, a length $L_{x}$ may be defined by the relation:

$$
L_{x}=\int_{0}^{\infty} R_{x} d x
$$

where $R_{x}$ is the correlation between the values of the component $u$ at two points separated by distance $x$ in the direction of the $x$ coordinate.

10. Isotropic turbulence. The simplest type of turbulence for theoretical or experimental investigation is that in which the intensity components in all directions are equal. More accurately, isotropic turbulence is defined by the condition that the mean value of any function of the velocity components and their derivatives at a given point is independent of rotation and reflection of the axes of reference. Changes in direction and magnitude of the fluctuations at a given point are wholly random and there is no correlation between the components of the fluctuations in different directions. Thus $\overline{u^{2}}=\overline{v^{2}}=\overline{w^{2}}$ and $\overline{u v}=\overline{v w}=\overline{u w}=0$.

There is a strong tendency toward isotropy in all turbulent motions. The turbulence at the center of a pipe in which the flow is eddying or in the natural wind at a sufficient height above the ground is approximately isotropic. A grid of round wires placed in a uniform fluid stream sets up a more or less regular eddy system of non-isotropic character which very quickly transforms into a field of uniformly distributed isotropic turbulence.

The assumption of isotropy introduces many simplifications in the statistical representation of turbulence. The two quantities, intensity and scale, appear to give a description of the statistical properties of the turbulent field 
which is sufficient for most purposes. Turbulent fields of this type can readily be produced experimentally and studied. The intensity may be varied from less than 0.1 to about 5.0 percent of the mean speed and the scale independently from a few $\mathrm{mm}$ to $25 \mathrm{~mm} .{ }^{14}$

11. Decay of isotropic turbulence. The kinetic energy of the turbulent fluctuations per unit volume is equal to $\frac{1}{2} \rho\left(\overline{u^{2}}+\overline{v^{2}}+\overline{w^{2}}\right)$ which for isotropic turbulence becomes $(3 / 2) \rho \overline{u^{2}}$. The rate of decay is therefore $-(3 / 2) \rho d\left(\overline{u^{2}}\right) / d t$. If the isotropic turbulence is superposed on a stream of uniform speed $U$, we may write $d t=d x / U$ and hence the rate of decay with respect to distance $x$ as $-(3 / 2) \rho U d\left(\overline{u^{2}}\right) / d x$.

In a fully developed turbulent flow the Reynolds stresses are proportional to the squares of the turbulent fluctuations. The work done against these stresses, which in the absence of external forces must come from the kinetic energy of the system, is proportional to $\rho u^{\prime 3} / L$ where $u^{\prime}$ is written for $\sqrt{u^{2}}$ and $L$ is a linear dimension defining the scale of the system, which may be taken as the $L$ defined by (9.2). Equating the two expressions for the dissipation and designating the constant of proportionality as $3 \mathrm{~A}$, we find:

$$
\text { - }(3 / 2)_{p} U d\left(u^{\prime 2}\right) / d x=3 A \rho u^{\prime 3} / L
$$

or

$$
L d\left(U / u^{\prime}\right) / d x=A .
$$

Integrating :

$$
U / u^{\prime}-U / u_{0}^{\prime}=A \int_{x_{0}}^{x} d x / L
$$

where $U / u_{0}^{\prime}$ is the value of $U / u^{\prime}$ at $x=x_{0}$. This equation has been found to give a very good representation of the experimental data. The essential features of the derivation were given by Taylor. To evaluate the in tegral, $L$ must be known as a function of $x$. Taylor's first proposal was to assume that $L$ is independent of $x$ and proportional to the mesh $M$ of the grid giving rise to the turbulence. If $L$ is constant,

$$
U / u^{\prime}-U / u_{0}^{\prime}=A\left(x-x_{0}\right) / L
$$

giving a linear variation of $U / u^{\prime}$ with $x$. Assuming $L / M=k$, Taylor found values of $A / k$ for data from various sources varying between 1.03 and 1.32.

${ }^{14}$ Dryden, H. L., Schubauer, G. B., Mock, W. C., Jr., and Skramstad, H. K., Measurements of intensity and scale of wind-tunnel turbulence and their relation to the critical Reynolds number of spheres, Tech. Rept. Nat. Adv. Comm. Aeron. No. 581 (1937). 
When measured values of $L$ became available it was found that $L$ increased as $x$ increased, the results being represented empirically within the accuracy of the measurements by the relation $L=L_{0}+c\left(x-x_{0}\right)$, whence

$$
U / u^{\prime}-U / u_{0}^{\prime}=(A / c) \log _{e}\left[1+c\left(x-x_{0}\right) / L_{0}\right] .
$$

Taylor ${ }^{15}$ found values of $A$ for data from various sources varying between 0.43 and 0.19 .

Further study suggests another relation for the variation of $L$ with $x$. A discussion of the general theory will be deferred until section 17 and the question discussed on purely dimensional considerations. If one assumes that $d u^{\prime} / d t$, the rate of change of intensity, and $d L / d t$, the rate of change of scale, are determined solely by the values of $L$ and $u^{\prime}$, i.e. that viscosity and upstream conditions have no influence, it follows from dimensional reasoning that

$$
L d\left(1 / u^{\prime}\right) / d t=A \quad \text { and } \quad\left(1 / u^{\prime}\right) d L / d t=B
$$

or

$$
L d\left(U / u^{\prime}\right) / d x=A \quad \text { and } \quad\left(U / u^{\prime}\right) d L / d x=B
$$

where $A$ and $B$ are numerical constants. The first equation of each pair is the same as equation (11.2); the second is a new relation.

Integration of equations (11.6) and (11.7) leads to the relations:

$$
\frac{u_{0}^{\prime}}{u^{\prime}}=\left[1+\frac{(A+B) u_{0}^{\prime}\left(x-x_{0}\right)}{L_{0} U}\right]^{A /(A+B)}
$$

and

$$
\frac{L}{L_{0}}=\left[1+\frac{(A+B) u_{0}^{\prime}\left(x-x_{0}\right)}{L_{0} U}\right]^{B /(A+B)}
$$

where $u_{0}^{\prime}$ and $L_{0}$ are the values at $x=0$.

If it is desired to introduce a reference dimension pertaining to the dimensions of the grid producing the disturbance, this may be done, but according to equations (11.8) and (11.9) any dimension may be used and the decay does not depend on its value. The mesh distance $M$ is of ten used but certain results reported by von Kármán ${ }^{16}$ show that if $M / d$ is not too small, the use

${ }_{15}$ Taylor, G. I., Some recent developments in the study of turbulence, Proc. Fifth Inter. Congr. Appl. Mech., Cambridge, Mass., 294 (1938). See later detailed report of measurements in Hall, A. A., Measurements of the intensity and scale of turbulence, Rept. and Memo. No. 1842, Aeronautical Research Committee, Great Britain (1938).

${ }^{16}$ Kármán, Th. von, Some remarks on the statistical theory of turbulence, Proc. Fifth Inter. Congr. Appl. Mech., Cambridge, Mass., 1938, p. 347. The grid dimensions are not given in 


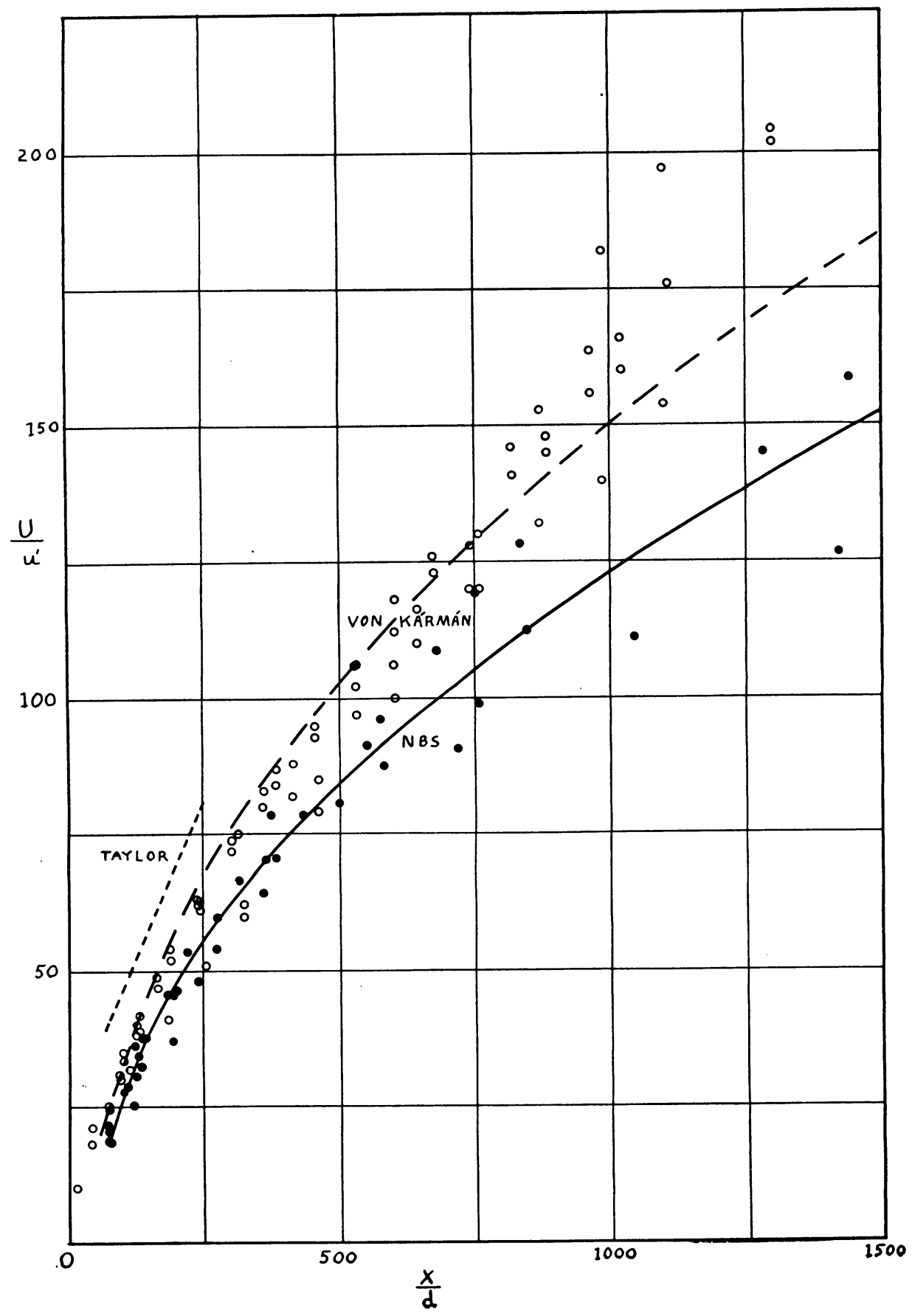

FIg. 1. The turbulent fluctuation $\boldsymbol{u}^{\prime}$ behind a grid of wires of diameter $d$ as a function of distance $x$ from the grid. 


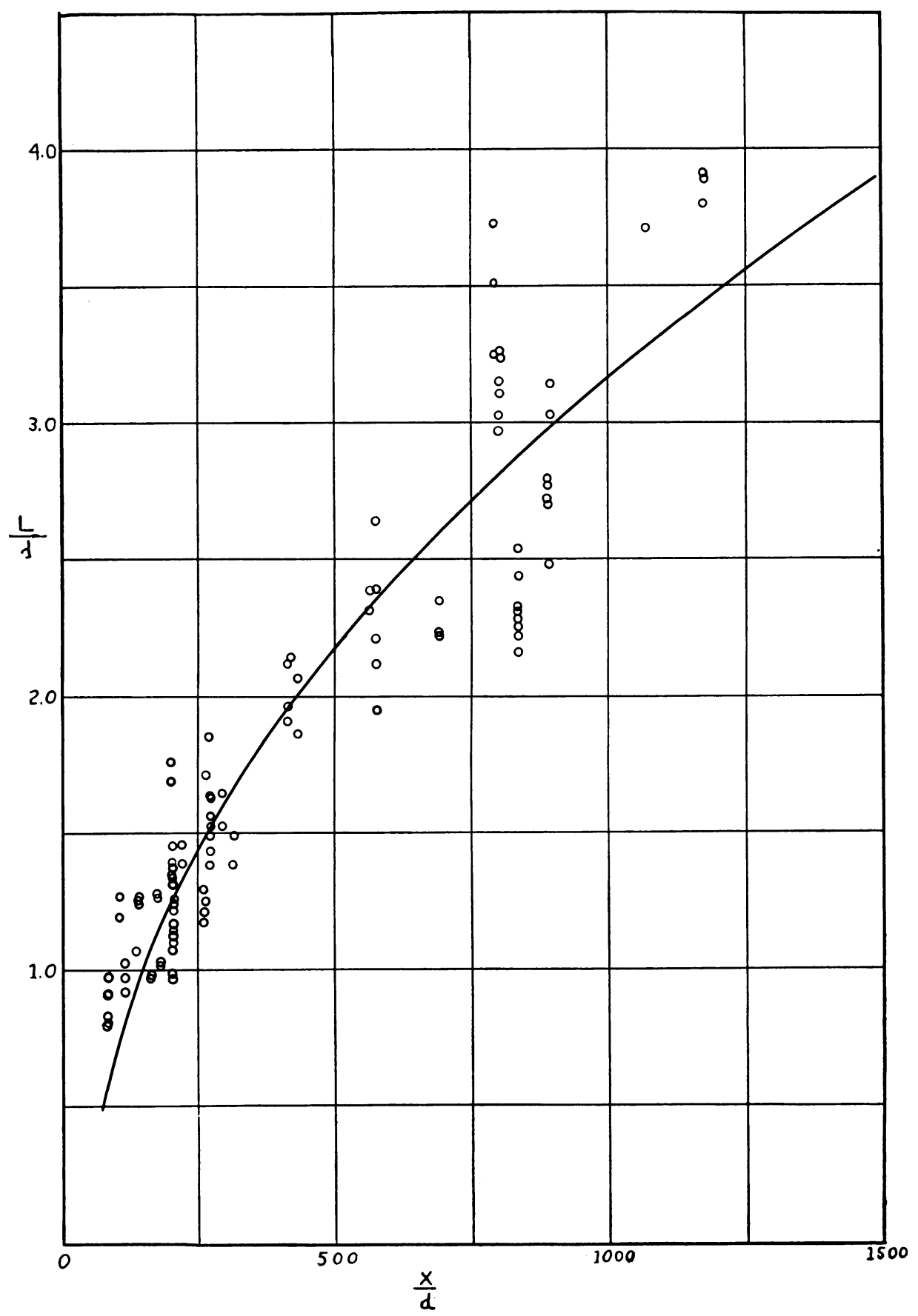

FIG. 2. The scale $L$ behind a grid of wires of diameter $d$ as a function of distance $x$ from the grid. 
of the wire diameter $d$ as the reference dimension leads to a single curve for all grids irrespective of the mesh-diameter ratio.

The available data are plotted in Figs. 1 and 2 from references in footnotes 14,15 , and 16 . The solid curves are respectively

and

$$
\left(U / u^{\prime}\right)^{2}=400[(1+0.04(x / d-80)]
$$

$$
(L / d)^{2}=0.264[(1+0.04(x / d-80)]
$$

which are in the form of equations (11.8) and (11.9) with the constants $A=B=0.2056$. These curves are frankly selected to fit the National Bureau of Standards data.

If one considers the complete system of screen and turbulent field, dimensional considerations suggest that for geometrically similar screens whose scale is fixed by some characteristic dimension, such as the mesh length $M$, the ratios $u^{\prime} / U$ and $L / M$ would be a function of $x / M$, of the Reynolds Number $U M / \nu$ and of the turbulence of the free stream $u_{t}^{\prime} / U$, in which the screen is placed. If the screens are not geometrically similar but are made up of cylindrical rods of diameter $d$, the intensity and scale also depend on $d / M$ and on the roughness of the screen. The effects of these parameters have not been fully investigated, and doubtless a part of the discrepancy between the available results is to be ascribed to the influence of these factors.

For example, the screens used at the National Bureau of Standards were either woven wire screens or wooden screens with fairly rough surfaces with the members interlacing in the wire screens and intersecting in the wooden screens. The ratio $d / M$ varied from 0.186 to 0.201 . The screens used by Hall were arranged in two planes, i.e., horizontal rods in one plane, vertical rods just touching the horizontal rods but in another plane. The ratio $d / M$ was 0.184 to 0.188 . Von Kármán has studied the effect of varying $d / M$ from 0.086 to 0.462 and has used screens both of the woven type (results published by von Kármán, loc. cit.) and of the biplane type (results not published). A study of these data suggests that the difference between the results for woven screens and biplane screens is unimportant and that if results are plotted in terms of $x / d$ rather than $x / M$ the effect of $d / M$ is small for values of $d / M$ near 0.2 . No data are available on the effect of roughness.

Few data are available on the effect of free stream turbulence. Hall obtained an increase of about 10 to 20 percent in $u^{\prime}$ for a 1 -inch screen at the

the paper, but Professor von Kármán has kindly supplied them as follows:

$\begin{array}{cccc}\text { Grid } & \begin{array}{c}\text { Mesh Distance, } M \\ \text { inches }\end{array} & \begin{array}{c}\text { Wire Diameter, } d \\ \text { inches }\end{array} & M / d \\ 1 & 4.96 & 0.230 & 2.16 \\ 2 & 5.00 & .105 & 4.75 \\ 3 & 5.07 & .084 & 6.03 \\ 4 & 4.99 & .043 & 11.6\end{array}$


same value of $x / M$ by increasing the free stream turbulence from 0.2 percent to 1.3 percent. We have had the opportunity of making some measurements behind the same 1-inch screen used in the measurements described in NACA Technical Report No. 581 in an airstream for which the free stream turbulence is 0.03 percent as compared with 0.85 percent for the older measurements. The results are shown in Fig. 3 as compared with Hall's measurements. It is obvious that the turbulence of the free stream is one of the controlling factors, but not the only one.

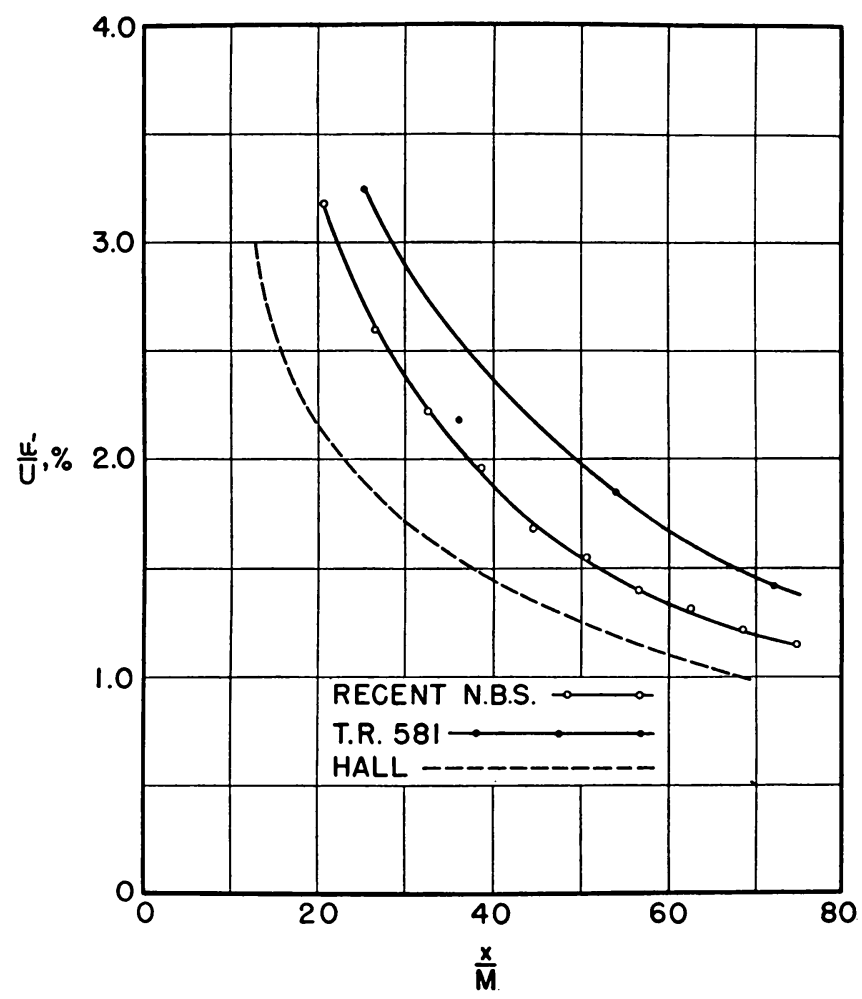

F1G. 3. Effect of free stream turbulence on the turbulence behind a 1 -inch screen.

The study of the turbulent field behind screens as affected by numerous parameters is of interest from the standpoint of a study of screens. However, the turbulent field may be regarded from another point of view, i.e. in relation solely to the theory of isotropic turbulence. If the turbulence is truly isotropic, and if its characteristics can be adequately described by the two quantities, intensity and scale, its behavior can depend only on the values of intensity and scale at some given point. The details of construction of the source screen and its distance upstream are of no importance. Even the influence of the turbulence of the free stream should be absorbed in the given 
values of $u^{\prime}$ and $L$ at some one point. The decay of isotropic turbulence is considered from this point of view in section 17 .

12. Effect of contraction. The behavior of turbulence in a contracting stream is of interest in connection with the flow in the entrance cone of a wind tunnel. Prandt1 ${ }^{17}$ suggested that the longitudinal components of the fluctuations were reduced in the ratio of 1 to $l$ where $l$ is the ratio of the entrance area to the exit area of the cone. This result was derived on the assumption that the gain in energy is the same for all filaments traversing the cone. The same result was obtained from the Helmholtz vortex theorem, which was also used to show that the lateral components were increased in the ratio $\sqrt{l}$. Since the mean speed increases proportional to $l$, the values of $u^{\prime} / U$ and $v^{\prime} / U$ are reduced according to this theory in the ratios $1 / l^{2}$ and $1 / \sqrt{l}$ respectively. This computation neglects the decay of the turbulence because of viscosity.

Taylor ${ }^{18}$ computed the effect of a contraction on certain mathematically defined forms of disturbance. Two objections may be offered to this treatment. First, as in Prandtl's treatment, the decay of the turbulence is neglected. Second, the computation is made on a regular disturbance which is assumed to retain its regularity. When the rapid development of an isotropic turbulent field from a Kármán vortex trail is considered, it is hard to believe that a regular vortex pattern could retain its character throughout the length of a wind tunnel entrance cone unless the scale was very large indeed.

If it is assumed that the istropic turbulent field is unaffected by changes in the mean speed, the decrease in $u^{\prime}$ may be computed from the decay during the time required for the fluid to traverse the cone. This time interval is $\int_{x_{0}}^{x_{1}} d x / U$. If $A$ is the area of the cross section at any value of $x, U A=U_{0} A_{0}$ where $U_{0}$ and $A_{0}$ are the values at $x=x_{0}$, and hence the time interval is $\int_{x_{0}}^{x_{1}} A d x / U_{0} A_{0}$.

There are as yet no suitable experimental data for checking any theory. In the measurements quoted by Taylor all the data were obtained sufficiently close to a grid to lie within the non-isotropic turbulence of the vortex trails from the individual wires.

13. The correlation tensor function. Von Kármán ${ }^{19}$ introduced the correlation tensor function in the statistical theory of turbulence as a generalization of the particular correlation coefficients discussed by Taylor. The correlation coefficients between any component of the speed fluctuation at a given point and any component of the speed fluctuation at another point

${ }_{17}$ Prandtl, L., Herstellung einwandfreier Luftströme (Windkanäle), Handbuch der Experimentalphysik, F. A. Barth, Leipzig, 1932, Vol. 4, Part 2, p. 73.

18 Taylor, G. I., Turbulence in a contracting stream, Z. angew. Math. u. Mech. 15, 91 (1935).

${ }^{19}$ Kármán, Th. von, ańd Howarth, L., On the statistical theory of isotropic turbulence, Proc. Roy. Soc. London, Ser. A, 164, 192 (1938). 
form a tensor. If one point is held fixed and the other varied, the tensor varies as a function of the coordinates of the variable point with respect to the fixed point. We may speak of this function as the correlation tensor function.

In isotropic turbulence the correlation tensor has spherical symmetry and the several components are functions only of the distance $r$ between the two points, and of the time $t$. Denote by $u_{1}, v_{1}, w_{1}$ and $u_{2}, v_{2}, w_{2}$ the components of the velocity fluctuations at the two points $\mathrm{P}_{1}$ and $\mathrm{P}_{2}$ having coordinates $\left(x_{1}, 0,0\right)$ and $\left(x_{2}, 0,0\right)$ respectively. Suppose that $\overline{u_{1}^{2}}, \overline{v_{1}^{2}}, \overline{w_{1}^{2}}$, which by isotropy are equal, are independent of position and equal to $\overline{u_{2}^{2}}$. Then $\overline{u_{2}^{2}}=\overline{v_{2}^{2}}=\overline{w_{2}^{2}}=\overline{u^{2}}$.

The correlation coefficients $\overline{v_{1} v_{2}} / \overline{u^{2}}$ and $\overline{w_{1} w_{2}} / \overline{u^{2}}$ will be identical because of isotropy and will be some particular function of the distance $r$ between

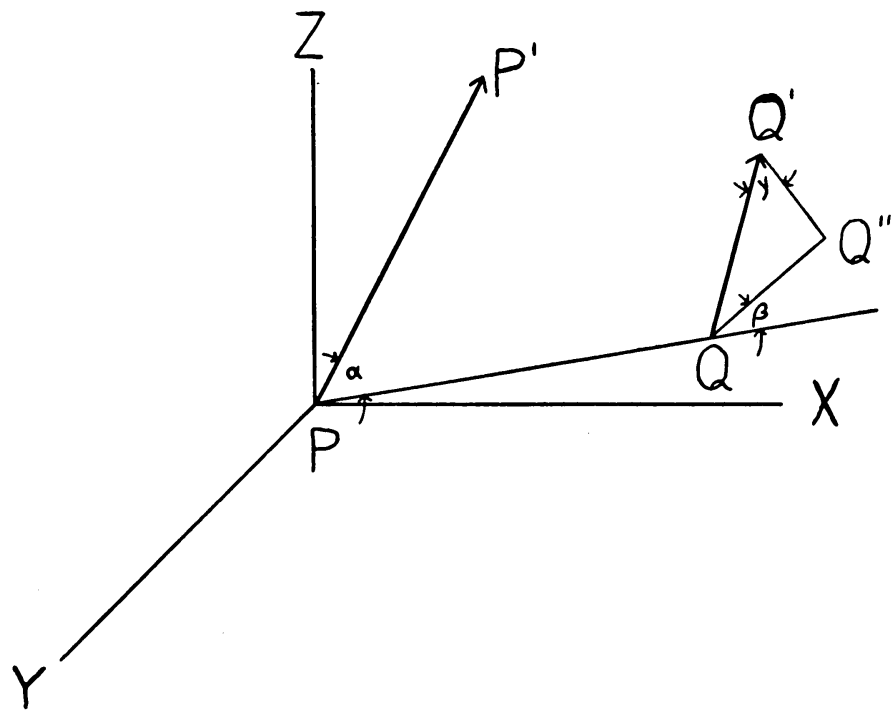

Fig. 4.

$\mathrm{P}_{1}$ and $\mathrm{P}_{2}$ and of the time $t$, say $g(r, t)$. The correlation coefficient $\overline{u_{1} u_{2}} / \overline{u^{2}}$ will also be a function of $r$ and $t$, say $f(r, t)$. The correlation coefficients $\overline{u_{1} v_{2}} / \overline{u^{2}}, \overline{u_{1} w_{2}} / \overline{u^{2}}, \overline{v_{1} u_{2}} / \overline{u^{2}}, \overline{v_{1} w_{2}} / \overline{u^{2}}, \overline{w_{1} u_{2}} / \overline{u^{2}}$ and $\overline{w_{1} v_{2}} / \overline{u^{2}}$ can be shown to be zero. Thus if the $Y$ and $Z$ axes are rotated about the $X$ axis through $180^{\circ}$, the absolute values of all components are unchanged but the signs of the $v$ and $w$ components are reversed. Denoting values referred to the new axes by capital letters, $U_{1}=u_{1}, U_{2}=u_{2}, V_{1}=-v_{1}, V_{2}=-v_{2}, W_{1}=w_{1}, W_{2}=-w_{2}$, so that, for example, $\overline{U_{1} V_{2}}=-\overline{u_{1} v_{2}}$. But by isotropy, the value of any function of the components is unchanged by rotation of the axes, and therefore $\overline{U_{1} V_{2}}=\overline{u_{1} v_{2}}$. To satisfy both relations $\overline{u_{1} v_{2}}$ must equal zero. Similarly for the other terms containing $u_{1}$ or $u_{2}$. By reflection in the $X Z$ plane $\overline{v_{1} w_{2}}$ and $\overline{w_{1} v_{2}}$ may likewise be shown to be zero. 
The correlation coefficient for components of the fluctuations in any arbitrary directions at any two points may be expressed in terms of the functions $f(r, t)$ and $g(r, t)$ and the geometrical
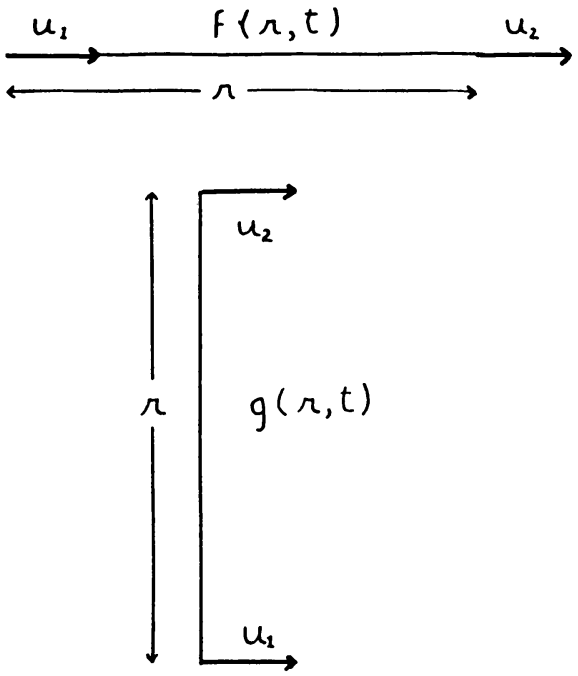

FIG. 5. The principal double correlations in isotropic turbulence.

parameters. Consider any two points $P$ and $Q$ and components of the fluctuations $p$ in the direction $\mathrm{PP}^{\prime}$ at $\mathrm{P}$ and $q$ in the direction $Q Q^{\prime}$ at $Q$. (Fig. 4). Denote by $Q Q^{\prime \prime}$ the orthogonal projection of $Q Q^{\prime}$ on the plane $P^{\prime} Q$; by $\alpha, \beta$, and $\gamma$ the angles $\mathrm{P}^{\prime} \mathrm{PQ}$, $\pi-\mathrm{PQQ}^{\prime \prime}$, and $\mathrm{QQ}^{\prime} \mathrm{Q}^{\prime \prime}$; and by $p_{1}, p_{2}$, $p_{3}$ and $q_{1}, q_{2}, q_{3}$ the components of the fluctuations at $P$ and $Q$ in the direction $P Q$, in the direction normal to $P Q$ and $Q^{\prime} Q^{\prime \prime}$, and in the direction $Q^{\prime \prime} Q^{\prime}$. Then

$$
\begin{aligned}
p= & p_{1} \cos \alpha+p_{2} \sin \alpha \\
q= & q_{1} \cos \beta \sin \gamma+q_{2} \sin \beta \sin \gamma \\
& +q_{3} \cos \gamma .
\end{aligned}
$$

Hence

$$
\overline{p q}=\overline{p_{1} q_{1}} \cos \alpha \cos \beta \sin \gamma+\overline{p_{2} q_{2}} \sin \beta \sin \alpha \sin \gamma
$$

the other terms vanishing as proved in the preceding paragraph. In terms of $f(r, t)$ and $g(r, t)$

$$
\overline{p q} / \overline{u^{2}}=[f(r, t) \cos \alpha \cos \beta+g(r, t) \sin \alpha \sin \beta] \sin \gamma .
$$

The correlations denoted by $f(r, t)$ and $g(r, t)$ are indicated in Fig. 5 .

If now any two points with coordinates $\left(x_{1}, y_{1}, z_{1}\right)$ and $\left(x_{2}, y_{2}, z_{2}\right)$ and speed fluctuations with components $u_{1}, v_{1}, w_{1}$ and $u_{2}, v_{2}, w_{2}$ are considered, the nine quantities $\overline{u_{1} u_{2}}, \overline{u_{1} v_{2}}, \overline{u_{1} w_{2}}, \overline{v_{1} u_{1}}, \overline{v_{1} v_{2}}, \overline{v_{1} w_{2}}, \overline{w_{1} u_{1}}, \overline{w_{1} v_{2}}$, and $\overline{w_{1} w_{2}}$ are the components of a second rank tensor. Each one may be evaluated by equation (13.2) in terms of $f(r, t)$ and $g(r, t)$ with the result in tensor notation

$$
\boldsymbol{R}=\frac{f(r, t)-g(r, t)}{r^{2}} \boldsymbol{r} \boldsymbol{r}+g(r, t) \boldsymbol{I}
$$

where $\boldsymbol{r}$ is the vector having components $X=x_{2}-x_{1}, Y=y_{2}-y_{1}, Z=z_{2}-z_{1}$ $r$ is $|r|$ and $I$ is the unit tensor $\left|\begin{array}{lll}1 & 0 & 0 \\ 0 & 1 & 0 \\ 0 & 0 & 1\end{array}\right|$. 
The velocity fluctuations satisfy the equation of continuity. Hence

$$
\frac{\partial u_{2}}{\partial x_{2}}+\frac{\partial v_{2}}{\partial y_{2}}+\frac{\partial w_{2}}{\partial z_{2}}=0 \text {. }
$$

Multiplying by $u_{1} / \overline{u^{2}}$ which is independent of $x_{2}, y_{2}, z_{2}$ and introducing the correlation coefficients $R_{u_{1} u_{2}}$, etc. and the components $X, Y, Z$ of $\boldsymbol{r}$ :

$$
\frac{\partial R_{u_{1} u_{2}}}{\partial X}+\frac{\partial R_{u_{1} v_{2}}}{\partial Y}+\frac{\partial R_{u_{1} w_{2}}}{\partial Z}=0 .
$$

From equation (13.4)

$$
R_{u_{1} u_{2}}=\frac{f-g}{r^{2}} X^{2}+g ; \quad R_{u_{1} v_{2}}=\frac{f-g}{r^{2}} X Y ; \quad R_{u_{1} w_{2}}=\frac{f-g}{r^{2}} X Z
$$

whence, remembering that $X^{2}+Y^{2}+Z^{2}=r^{2}, \partial r / \partial X=X / r, \partial f / \partial X=(\partial f / \partial r)$ $(\partial r / \partial X)=(X / r)(\partial f / \partial r)$, etc., equation (13.6) becomes

$$
X[2(f-g)+r(\partial f / \partial r)]=0 .
$$

The continuity equation must be true for any value of $X$. Hence

$$
2 f(r, t)-2 g(r, t)=-r \partial f(r, t) / \partial r .
$$

The correlation tensor can thus be expressed in terms of a single scalar function, either $f(r, t)$ or $g(r, t)$. The function $g(r, t)$ is the correlation coefficient previously denoted by $R_{y}$. The scale $L=\int_{0}^{\infty} R_{y} d y=\int_{0}^{\infty} g d r$. The integral $\int_{0}^{\infty} R_{x} d x=\int_{0}^{\infty} f d r$ is termed the longitudinal scale $L_{x}$ to distinguish it from the lateral scale $L$. Obviously from equation (13.8)

$$
L-L_{x}=\frac{1}{2} \int_{0}^{\infty} r(\partial f / \partial r) d r=\frac{1}{2} \int_{0}^{\infty} x\left(\partial R_{x} / \partial x\right) d x .
$$

Since $f$ and $g$ are even functions of $r$,

$$
\begin{aligned}
& f=1+f_{0}^{\prime \prime} r^{2} / 2+\cdots \\
& g=1+g_{0}^{\prime \prime} r^{2} / 2+\cdots .
\end{aligned}
$$

From equation (13.8), $2 f_{0}^{\prime \prime}=g_{0}^{\prime \prime}$, whence for small values of $r$,

$$
\begin{aligned}
R & =\left[1+\left(g_{0}^{\prime \prime} / 2\right) r^{2}\right] I+\left[\left(f_{0}^{\prime \prime}-g_{0}^{\prime \prime} / 2\right]_{r \boldsymbol{r}}\right. \\
& =\left(1+f_{0}^{\prime \prime} r^{2}\right) I-(1 / 2) f_{0}^{\prime \prime} r \boldsymbol{r} .
\end{aligned}
$$

We require later the second derivatives of $R$ at $r=0$, i.e. $X=Y=Z=0$, as follows:

$$
\frac{\partial^{2} R_{u_{1} u_{2}}}{\partial X^{2}}=\frac{\partial^{2} R_{v_{1} v_{2}}}{\partial Y^{2}}=\frac{\partial^{2} R_{w_{1} w_{2}}}{\partial Z^{2}}=f_{0}^{\prime \prime}
$$


$\frac{\partial^{2} R_{u_{1} u_{2}}}{\partial Y^{2}}=\frac{\partial^{2} R_{u_{1} u_{2}}}{\partial Z^{2}}$ and similar terms obtained by cyclic exchange $=2 f_{0}^{\prime \prime}$

$\frac{\partial^{2} R_{u_{1} v_{2}}}{\partial X \partial Y}$ and similar terms obtained by cyclic exchange $=-(1 / 2) f_{0}^{\prime \prime}$.

All others, e.g. $\frac{\partial^{2} R_{u_{1} v_{2}}}{\partial X \partial Z}$ etc. are zero.

Von Kármán points out that the correlation tensor is of the same form as the stress tensor for a continuous medium when there is spherical symmetry. In the analogy $f(r)$ corresponds to the principal radial stress at any point, $g(r)$ to the principal transverse stress, and the several $R$ 's to the stress components over planes normal to the coordinate axes. The relation between $f$ and $g$ given by the continuity equation corresponds to the condition for equilibrium of the stresses.

Equation (13.8) has been experimentally checked at the National Physical Laboratory. ${ }^{16}$

14. Correlation between derivatives of the velocity fluctuations. In further developments it will be necessary to know the mean values of the products of the derivatives of the components of the fluctuations at a given point, for example $\left(\overline{\left.\partial u_{1} / \partial x_{1}\right)\left(\partial v_{1} / \partial y_{1}\right.}\right)$. These mean values may readily be computed from the correlation tensor. Thus:

$$
\frac{\partial\left(\overline{u_{1} v_{2}}\right)}{\partial x_{1}}=\overline{u^{2}} \frac{\partial\left(R_{u_{1} v_{2}}\right)}{\partial x_{1}}=-\overline{u^{2}} \frac{\partial\left(R_{u_{1} v_{2}}\right)}{\partial X} .
$$

Since $v_{2}$ is not a function of $x_{1}$, this may be written

$$
\overline{\left(\partial u_{1} / \partial x_{1}\right) v_{2}}=-\overline{u^{2}} \partial R_{u_{1} v_{2}} / \partial X .
$$

Differentiating now with respect to $y_{2}$

$$
\overline{\frac{\partial u_{1}}{\partial x_{1}} \frac{\partial v_{2}}{\partial y_{2}}}=\frac{\partial}{\partial y_{2}}\left(\overline{\frac{\partial u_{1}}{\partial x_{1}} v_{2}}\right)=-\overline{u^{2}} \frac{\partial}{\partial y_{2}}\left(\frac{\partial R_{u_{1} v_{2}}}{\partial X}\right)=-\overline{u^{2}} \frac{\partial^{2} R_{u_{1} v_{2}}}{\partial X \partial Y} .
$$

Now letting $P_{1}$ and $P_{2}$ coincide,

$$
\overline{\frac{\partial u}{\partial x} \frac{\partial v}{\partial y}}=-\overline{u^{2}}\left(\frac{\partial^{2} R_{u v}}{\partial X \partial Y}\right) X=Y=0 .
$$

The limiting value of the second derivative has previously been computed (equation (13.15)), whence

$$
\overline{\frac{\partial u}{\partial x} \frac{\partial v}{\partial y}}=\frac{f_{0}^{\prime \prime}}{2} \overline{u^{2}} .
$$

By similar reasoning it may be shown that 


$$
\begin{aligned}
\overline{\left(\frac{\partial u}{\partial x}\right)^{2}}=\overline{\left(\frac{\partial v}{\partial y}\right)^{2}} & =\overline{\left(\frac{\partial w}{\partial z}\right)^{2}}=-\overline{u^{2}} f_{0}^{\prime \prime} \\
\overline{\left(\frac{\partial u}{\partial y}\right)^{2}}=\overline{\left(\frac{\partial u}{\partial z}\right)^{2}} & =\overline{\left(\frac{\partial v}{\partial x}\right)^{2}}=\overline{\left(\frac{\partial v}{\partial z}\right)^{2}}=\overline{\left(\frac{\partial w}{\partial x}\right)^{2}} \\
& =\overline{\left(\frac{\partial w}{\partial y}\right)^{2}}=-2 \overline{u^{2}} f_{0}
\end{aligned}
$$

and

$$
\overline{\frac{\partial v}{\partial x} \frac{\partial u}{\partial y}}=\overline{\frac{\partial w}{\partial y} \frac{\partial v}{\partial z}}=\overline{\frac{\partial u}{\partial z} \frac{\partial w}{\partial x}}=\frac{\overline{u^{2}}}{2} f_{0}^{\prime \prime} .
$$

The method can be extended to derivatives of higher order.

15. Triple correlations. Von Kármán designates the mean values of the product of three components of the velocity fluctuations, two of which are taken at one arbitrary point and the third at a second arbitrary point, as triple correlations. They arise when correlation coefficients are introduced into the equations of motion. He shows that the triple correlations are components of a tensor of third rank designated $T$ which is a function of $X, Y, Z$ and the time. He proves that in isotropic turbulence this tensor can be expressed in terms of three functions $h(r, t)$, $k(r, t)$, and $q(r, t)$ corresponding to
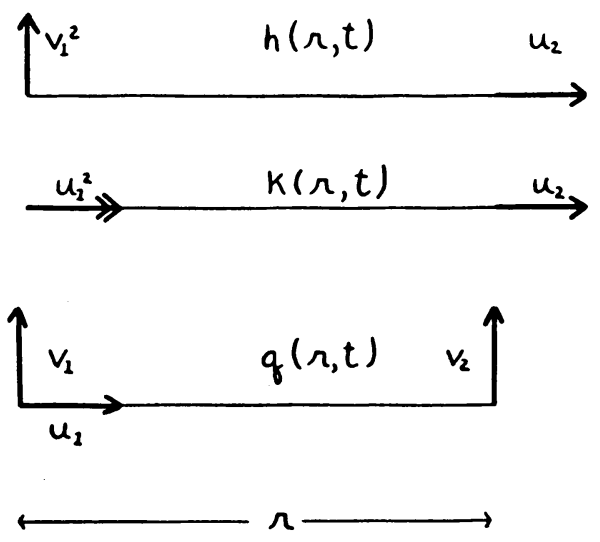

FIG. 6. The principal triple correlations in isotropic turbulence. the correlations shown in Fig. 6, and that the development of these functions in powers of $r$ begins with the $r^{3}$ term. The equation of continuity permits the expression of $k$ and $q$ in terms of $h$ by the relations:

$$
\begin{aligned}
& k=-2 h \\
& q=-h-(r / 2)(d h / d r) .
\end{aligned}
$$

Thus the tensor $T$ can be expressed in terms of a single scalar function $h(r, t)$.

16. Propagation of the correlation with time. The fluctuations are assumed to satisfy the equations of motion, namely,

$$
\begin{aligned}
& \frac{\partial u_{1}}{\partial t}+u_{1} \frac{\partial u_{1}}{\partial x_{1}}+v_{1} \frac{\partial u_{1}}{\partial y_{1}}+w_{1} \frac{\partial u_{1}}{\partial z_{1}} \\
&=-\frac{1}{\rho} \frac{\partial p}{\partial x_{1}}+\nu\left(\frac{\partial^{2} u_{1}}{\partial x_{1}^{2}}+\frac{\partial^{2} u_{1}}{\partial y_{1}^{2}}+\frac{\partial^{2} u_{1}}{\partial z_{1}^{2}}\right)
\end{aligned}
$$


and the two equations obtained by cyclic permutation.

Multiplying this equation by $u_{2}$, introducing $X, Y$, and $Z$, and taking mean values:

$$
\begin{aligned}
\overline{u_{2} \frac{\partial u_{1}}{\partial t}}-\frac{\partial \overline{\left(u_{1}^{2} u_{2}\right)}}{\partial X} & -\frac{\partial\left(\overline{u_{1} v_{1} u_{2}}\right)}{\partial Y}-\frac{\left.\partial \overline{\left(u_{1} w_{1} u_{2}\right.}\right)}{\partial Z} \\
= & -\frac{1}{\rho} \overline{u_{2} \frac{\partial p}{\partial x_{1}}}+\nu\left(\frac{\partial^{2} u_{1} u_{2}}{\partial X^{2}}+\frac{\partial^{2} u_{1} u_{2}}{\partial Y^{2}}+\frac{\partial^{2} u_{1} u_{2}}{\partial Z^{2}}\right) .
\end{aligned}
$$

By an analogous procedure, it may be shown that:

$$
\begin{aligned}
& \overline{u_{1} \frac{\partial u_{2}}{\partial t}}-\frac{\overline{\partial u_{2}^{2} u_{1}}}{\partial X}-\frac{\overline{\partial u_{2} v_{2} u_{1}}}{\partial Y}-\frac{\overline{\partial u_{2} w_{2} u_{1}}}{\partial Z} \\
& =-\frac{1}{\rho} \overline{u_{1} \frac{\partial p}{\partial x_{2}}}+\nu\left(\frac{\partial^{2} u_{1} u_{2}}{\partial X^{2}}+\frac{\partial^{2} u_{1} u_{2}}{\partial Y^{2}}+\frac{\partial^{2} u_{1} u_{2}}{\partial Z^{2}}\right) .
\end{aligned}
$$

Von Kármán shows that the pressure terms vanish. Adding the two equations and introducing the correlation coefficients, we find

$$
\begin{aligned}
\frac{\partial}{\partial t}\left(\overline{u^{2}} R_{u_{1} u_{2}}\right) & \left.\left.-\left(\overline{u^{2}}\right)^{3 / 2} \frac{\partial}{\partial X} \overline{\left(\overline{u_{1}^{2} u_{2}}\right.}+\overline{u_{2}^{2} u_{1}}\right)-\overline{\left(u^{2}\right.}\right)^{3 / 2} \frac{\partial}{\partial Y}\left(\overline{u_{1} v_{1} u_{2}}+\overline{u_{2} v_{2} u_{1}}\right) \\
& -\left(\overline{u^{2}}\right)^{3 / 2} \frac{\partial}{\partial Z}\left(\overline{u_{1} w_{1} u_{2}}+\overline{\left.u_{2} w_{2} u_{1}\right)}\right. \\
= & 2 \nu \overline{u^{2}}\left[\frac{\partial^{2} \overline{u_{1} u_{2}}}{\partial X^{2}}+\frac{\partial^{2} \overline{u_{1} u_{2}}}{\partial Y^{2}}+\frac{\partial^{2} \overline{u_{1} u_{2}}}{\partial Z^{2}}\right] .
\end{aligned}
$$

This equation may be expressed in terms of the functions $f, g, k, q$ and $h$. Then by using the relations (15.1) and (15.2) between these functions obtained from the equation of continuity, a partial differential equation between $f$ and $h$ is obtained, namely

$$
\frac{\partial\left(f \overline{\left.u^{2}\right)}\right.}{\partial t}+2\left(\overline{u^{2}}\right)^{3 / 2}\left(\frac{\partial h}{\partial r}+\frac{4 h}{r}\right)=2 \nu \overline{u^{2}}\left(\frac{\partial^{2} f}{\partial r^{2}}+\frac{4}{r} \frac{\partial f}{\partial r}\right) .
$$

This is the equation for the change of the function $f$ with time, but it cannot be solved without some knowledge of the function $h$.

17. Self-preserving correlation functions. Let us suppose that the functions $f(r, t)$ and $h(r, t)$ preserve the same form as $t$ increases, only the scale varying. Such functions will be termed "self-preserving." If $L$ is some measure of the scale of the correlation curve, $f$ and $h$ will be functions of $r / L$ only, where $L$ is a function of $t$. The length $L$ may be any measure of the scale such 
as the radius of curvature of the correlation curve at $r=0$ or any other designated point, the value of $r$ for a given value of the correlation coefficient, or the quantity obtained by integration of the correlation coefficient from $r=0$ to infinity which has previously been termed the scale of the turbulence. Introducing the new variable $\psi=r / L$ and placing $\left(\overline{u^{2}}\right)^{1 / 2}=u^{\prime}$ in equation (16.5), we obtain

$$
\frac{f L}{u^{\prime 3}} \frac{d u^{\prime 2}}{d t}-\frac{1}{u^{\prime}} \frac{d L}{d t} \psi \frac{\partial f}{\partial \psi}+2\left(\frac{\partial h}{\partial \psi}+4 \frac{h}{\psi}\right)=\frac{2}{N}\left(\frac{\partial^{2} f}{\partial \psi^{2}}+\frac{4}{\psi} \frac{\partial f}{\partial \psi}\right)
$$

where $N$ is the Reynolds Number of the turbulence $u^{\prime} L / \nu$. Since the coefficient of the third term is a numerical constant, the functions $f$ and $h$ will be functions of $\psi$ and $t$ alone only if the coefficients of the other terms are also numerical constants. This requires that

$$
\begin{aligned}
\frac{L}{u^{\prime 3}} \frac{d u^{\prime 2}}{d t} & =-L \frac{d\left(1 / u^{\prime}\right)}{d t}=-A \\
\frac{1}{u^{\prime}} \frac{d L}{d t} & =B \\
\frac{u^{\prime} L}{\nu} & =N_{0}
\end{aligned}
$$

where $A, B$, and $N_{0}$ are independent of $u^{\prime}, L$, and $t$. It is readily shown that these relations are consistent only if $A=B$ and that the solutions are

$$
\begin{aligned}
\frac{1}{u^{\prime 2}}-\frac{1}{u_{0}^{\prime 2}} & =\frac{2 A}{N_{0} \nu} t \\
L^{2}-L_{0}^{2} & =2 A N_{0} \nu t
\end{aligned}
$$

where $u_{0}^{\prime}$ and $L_{0}$ are the values for $t=0$ and $u_{0}^{\prime} L_{0} / \nu=N_{0}$.

These equations are in the form of equations (11.8) and (11.9) with $A=B$ and agree well with the formulation of the experimental data represented by equations (11.10) and (11.11) with $2 A N_{0} \nu / L_{0}^{2}=2 A u_{0}^{2} / N_{0} \nu=2 A \sqrt{\overline{u_{0}^{2}}} / L_{0}=0.04$, corresponding to $t=0$ at a distance of 80 wire diameters from the grid. The constant $A$ is equal to 0.2056 when $L$ is defined as $\int_{0}^{\infty} R_{y} d y$.

For self-preserving turbulence equation (16.5) becomes

$$
-A f-A \psi(\partial f / \partial \psi)+2(\partial h / \partial \psi+4 h / \psi)=\left(2 / N_{0}\right)\left[\left(\partial^{2} f / \partial \psi^{2}+(4 / \psi)(\partial f / \partial \psi)\right] .\right.
$$

This equation determines the shape of the correlation curve. Von Kármán ${ }^{19}$ discusses the shape when the function $h$ is neglected. The shape depends on the Reynolds Number $N_{0}$ of the turbulence. The shape also depends on the constant $A$ but closer examination shows that $A$ is always associated with $L$ 
and is dependent on the method of defining $L$. If $\psi$ is set equal to $r A / L$ instead of $r / L$, and the length $L / A$ is used instead of $L$ in the definition of the Reynolds Number of the turbulence, the $A$ disappears from equation (17.7). Whether the values of $L$ defined by $\int g d r$ will yield the same values of $A$ for all shapes of correlation curves described by (17.7) cannot be definitely answered.

Approximate solutions of (17.7) are not easy since it turns out that $f$ varies with $N_{0}$ in such a manner that, for small values of $\psi$ at least, the term on the right-hand side is of the order of unity.

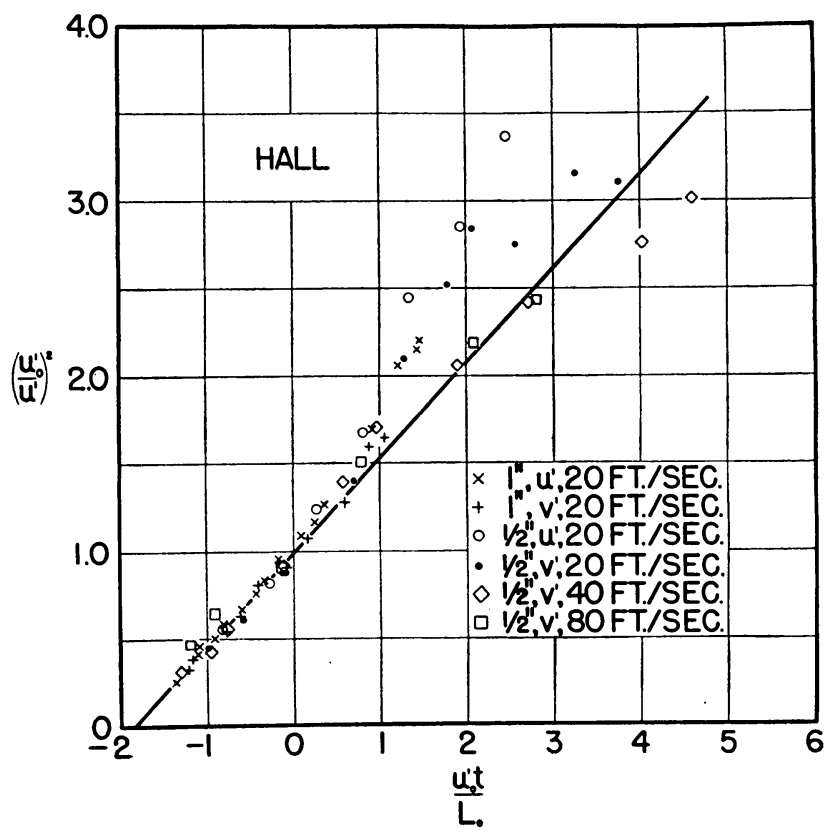

Fig. 7. Hall's measurements of turbulence behind screens.

According to this suggested theory, the shape is self-preserving and the Reynolds Number remains constant during the decay of a given turbulent field. The scale approaches very large values as the intensity approaches very small values. The length $\lambda$ (which is discussed in section 18) is proportional to $L$. For different values of the Reynolds Number of the turbulence the constant of proportionality varies inversely as the square root of the Reynolds Number. Likewise the shape of the correlation curve varies with the Reynolds number of the turbulence.

Equation (17.6) shows the same functional relation between the scale and the time as given by Prandtl at the Turbulence Symposium as a result of his analysis of photographs of the decay of isotropic turbulence. 
Von Kármán also discusses the case in which the assumption is made that the self-preserving feature applies only to large values of $\psi$ and the Reynolds Number $N_{0}$ is sufficiently large that the right-hand term of (17.7) can be neglected. In this case (17.2) and (17.3) are obtained without (17.4) and the solution is identical with that given by (11.8) and (11.9) of section 11. The theoretical equations (17.5) and (17.6) do not involve either $U$

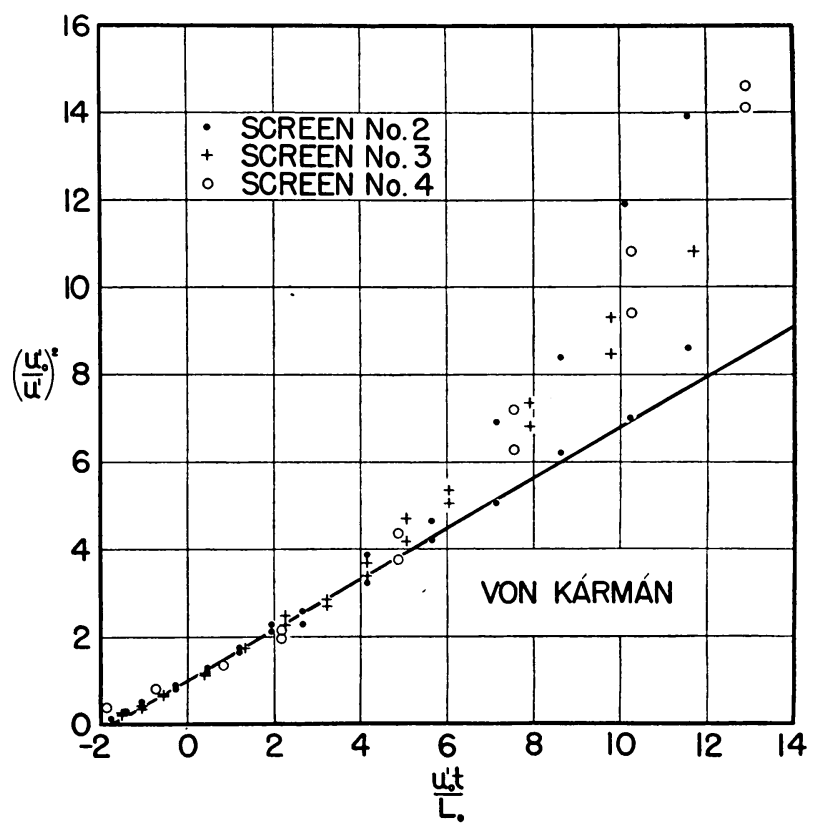

FIG. 8. Von Kármán's measurements of turbulence behind screens.

or $M$ explicitly. However, for comparison with experimental data, they may be written as follows:

$$
\begin{gathered}
\left(\frac{u_{0}^{\prime}}{u^{\prime}}\right)^{2}=1+\frac{2 A u_{0}^{\prime} t}{L_{0}}=1+2 A \frac{u_{0}^{\prime}}{U} \frac{\left(x-x_{0}\right)}{L_{0}} \\
\left(\frac{L}{L_{0}}\right)^{2}=1+\frac{2 A u_{0}^{\prime} t}{L_{0}}=1+2 A \frac{u_{0}^{\prime}}{U} \frac{\left(x-x_{0}\right)}{L_{0}} .
\end{gathered}
$$

Both $u_{0}^{\prime}$ and $L_{0}$ should be known, but unfortunately $L_{0}$ was not measured in all of the experiments.

Figures 7, 8, and 9 show the results of Hall, of von Kárman, and of the author and his associates (designated NBS) plotted in a manner to facilitate comparison with equation (17.8).

The reference position $x_{0}$ has been taken as 40 times the mesh length ex- 
cept for von Kármán's results for which $x_{0}$ was taken as 212.5 times the rod diameter (equivalent to $x_{0} / M=40$ for $d / M=0.188$ ). In the absence of definite information as to $L_{0}, L_{0} / M$ was assumed equal to 0.29 except for von Kármán's results for which $L_{0} / d$ was assumed to be 1.54 (equivalent to $L_{0} / M=0.29$ for $d / M=0.188$ ). The value of $u_{0}^{\prime}$ was determined by interpolation from the observations of each experimenter near $x / M=40$, giving the following results.

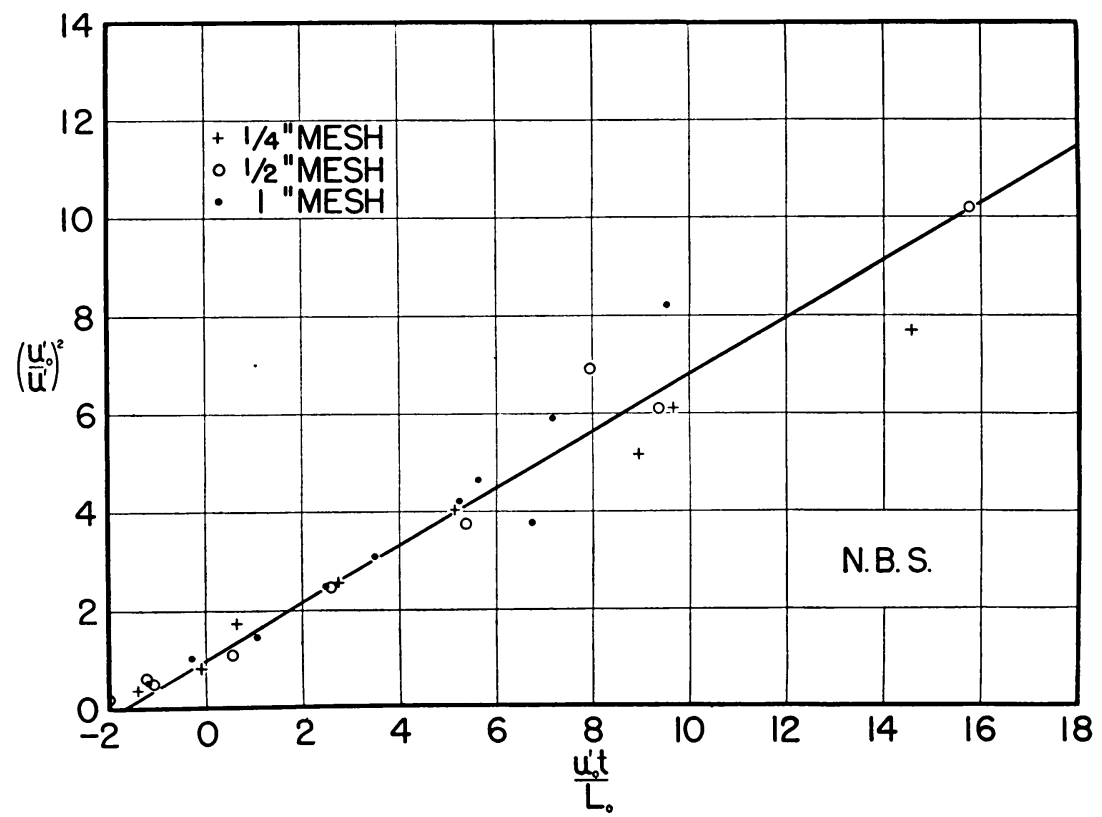

FIG. 9. NBS measurements of turbulence behind screens.

$\begin{array}{lccccc}\text { Experimenter } & \begin{array}{c}\text { Mesh } \\ \text { Inches }\end{array} & \begin{array}{c}\text { Rod } \\ \text { Diameter } \\ \text { Inches }\end{array} & \begin{array}{c}\text { Air Speed } \\ \text { ft/sec }\end{array} & u_{0}^{\prime} / U & \text { Remarks } \\ \text { Hall } & 1.0 & 0.188 & 20 & 0.0146 & \\ & 0.5 & .092 & 20 & .0144 & \\ & 0.5 & .092 & 40 & .0152 & \\ & 0.5 & .092 & 80 & .0174 & \\ \text { von Kármán } & 0.5 & 0.105 & 38 \text { and } 54 & .0201 & \text { Screen 2 } \\ & 0.5 & .084 & 38 \text { and } 75 & .0201 & \text { Screen 3 } \\ & 0.5 & .043 & 38 \text { and } 75 & .0299 & \text { Screen 4 } \\ \text { NBS } & & & & & \\ & 0.25 & 0.050 & 20-70 & 0.0250 & \text { NACA Tech. Rept. 581 } \\ & 0.5 & .096 & 20-70 & .0221 & \text { NACA Tech. Rept. 581 } \\ & 1.0 & .196 & 20-70 & .0224 & \text { NACA Tech. Rept. 581 } \\ & & & & & \\ & 1.0 & 0.196 & 30 & 0.0188 & \text { Recent tests } \\ & 1.0 & .196 & 70 & .0173 & \text { Recent tests }\end{array}$


The values of $u_{0}^{\prime} / U$ range from 0.0144 to 0.0299 ; presumably the differences are due mainly to the factors discussed in section 11 , although systematic errors may be partly responsible.

In each figure, equation (17.8) with constant $A$ equal to 0.29 is plotted as a straight line. Most of the points would be better fitted by a curve of increasing slope with increasing time. It thus appears that equation (11.9) with $(A+B) / A$ having some value between 1 and 2 fits the experimental data better than (17.8).

However, the data are not at all consistent. The departures are largest for the smaller values of $u^{\prime} / U$. In Hall's experiments, the results on the $\frac{1}{2}$-inch screen show little systematic departure at 40 and $80 \mathrm{ft} / \mathrm{sec}$, whereas those on the same screen at $20 \mathrm{ft} / \mathrm{sec}$ and on the 1 -inch screen begin to rise above the line at $u_{0}^{\prime} t / L_{0}=0.5$. Von Kármán's data on screen 2 at $38 \mathrm{ft} / \mathrm{sec}$ lie near the line; those on the same screen at $54 \mathrm{ft} / \mathrm{sec}$ and on screens 3 and 4 at 38 and $75 \mathrm{ft} / \mathrm{sec}$ begin to rise above the line at $u_{0}^{\prime} t / L_{0}=4.0$. The older results of the author and his associates, while scattered, agree with the line within 12 percent to $u_{0}^{\prime} t / L_{0}=18$; the more recent results begin to rise above the line at $u_{0}^{\prime} t / L_{0}=0.5$ and are in fair agreement with Hall's data on a 1-inch screen. Unfortunately, data at large values of $x / M$ could not be obtained in the recent experiments.

Thus, even when attention is confined to the behavior of the isotropic turbulent field, there remain discrepancies in the experimental data such that no definite conclusions can be drawn as to the merits of any theory. Further experiments are required under carefully controlled conditions in an air stream of low turbulence over a wide range of values of $x / M$ and with due regard to the various systematic errors that may be present. These experiments would be of the greatest value if the scale were also measured.

18. The length $\lambda$. Relation between $\lambda$ and $L$. The general expression for the mean rate of dissipation in the flow of a viscous fluid is:

$$
\begin{aligned}
W=\mu\left\{2 \overline{\left(\frac{\partial u}{\partial x}\right)^{2}}+2 \overline{\left(\frac{\partial v}{\partial y}\right)^{2}}+2 \overline{\left(\frac{\partial w}{\partial z}\right)^{2}}+\overline{\left(\frac{\partial v}{\partial x}+\frac{\partial u}{\partial y}\right)^{2}}\right. \\
+\overline{\left.\left(\frac{\partial w}{\partial y}+\frac{\partial v}{\partial z}\right)^{2}+\left(\frac{\partial u}{\partial z}+\frac{\partial w}{\partial x}\right)^{2}\right\}}
\end{aligned}
$$

where $\mu$ is the viscosity.

For isotropic turbulence this becomes:

$$
\frac{W}{\mu}=6 \overline{\left(\frac{\partial u}{\partial x}\right)^{2}}+6 \overline{\left(\frac{\partial u}{\partial y}\right)^{2}}+6 \overline{\frac{\partial v}{\partial x} \frac{\partial u}{\partial y}}
$$

which, from the relations given in section 14 , reduces to:

$$
W=-7.5 \mu_{-}^{2} \overline{u^{2}} g_{0}^{\prime \prime}=7.5 \mu \overline{(\partial u / \partial y)^{2}} .
$$


But $g_{0}^{\prime \prime}$ is defined by:

$$
g_{0}^{\prime \prime}=-2 \lim _{r \rightarrow 0}\left(\frac{1-g}{r^{2}}\right)
$$

and has the dimensions of the reciprocal of the square of a length. Let

$$
g_{0}^{\prime \prime}=-2 / \lambda^{2}
$$

the factor 2 being introduced to conform to Taylor's definition of $\lambda$. Then

$$
W=15 \mu \overline{u^{2}} / \lambda^{2} \text {. }
$$

The length $\lambda$ may be interpreted in several ways. Equation (18.6) may be considered a definition, $\lambda$ being regarded roughly as a measure of the diameters of the smallest eddies which are responsible for the dissipation of energy. Or, since $1 / \lambda^{2}=\lim _{r \rightarrow 0}(1-g) / r^{2}=\lim _{Y \rightarrow 0}\left(1-R_{y}\right) Y^{2}, \lambda^{2}$ is a measure of the radius of curvature of the $R_{y}$ curve at $Y=0$. Or, if a parabola is drawn tangent to the $R_{y}$ curve at $Y=0$, this parabola cuts the axis at the point $Y=\lambda$.

Since $W=-(3 / 2) \rho\left(d \overline{u^{2}} / d t\right)$, the decay law may be written:

$$
d \overline{u^{2}} / d t=-10 \nu \overline{u^{2}} / \lambda^{2} .
$$

This result can also be derived directly from equation (16.5) as shown by von Kármán.

By comparing this expression for the decay law with that previously given (equation 17.2), namely

$$
d \overline{u^{2}} / d t=-A u^{\prime 3} / L
$$

it is seen that

$$
A u^{\prime} / L=10 \nu / \lambda^{2}
$$

or, since $u^{\prime} L / \nu=N_{0}$

$$
\lambda^{2} / L^{2}=10 / A N_{0}
$$

Introducing the experimental value of $A$,

$$
\lambda / L=6.97 / \sqrt{N_{0}} .
$$

A similar relation holds for $\lambda / L_{x}$ where $L_{x}$ is the longitudinal scale. If the Reynolds Number is formed from $L_{x}$, the numerical constant is approximately 4.93 .

During the decay of self-preserving turbulence $N_{0}$ is constant and $\lambda$ is proportional to $L$ but the constant of proportionality varies inversely as $\sqrt{N_{0}}$ for turbulent fields of different Reynolds Number.

Although it cannot be expected on physical grounds that these relations hold at very low values of $N_{0}$, there is no experimental evidence of any de- 
parture from equations (18.7) and (18.8) for values of $N_{0}$ as low as 10. There seems to be no difficulty in drawing correlation curves for which $\lambda$ is greater than $L$, but no such experimental curves have been measured. However, in an example quoted by Taylor, ${ }^{15} \lambda / L$ is as great as 0.86 .

19. The spectrum of turbulence, relation between spectrum and correlation. The description of turbulence in terms of intensity and scale resembles the description of the molecular motion of a gas by temperature and mean free path. A more detailed picture can be obtained by considering the distribution of energy among eddies of different sizes, or more conveniently the distribution of energy with frequency. Just as a beam of white light may be separated into a spectrum by the action of a prism or grating, the electric current produced by a hot wire anemometer subjected to the speed fluctuations may be analyzed by means of electric filters into a spectrum.

The mean value of $\overline{u^{2}}$ may be regarded as made up of a sum of contributions $\overline{u^{2}} F(n) d n$, where $F(n)$ is the contribution from frequencies between $n$ and $n+d n$ and $\int_{0}^{\infty} F(n) d n=1$. The curve of $F(n)$ plotted against $n$ is the spectrum curve. According to the proof given by Rayleigh and quoted by Taylor ${ }^{20}$

$$
F(n)=2 \pi^{2} \lim _{T \rightarrow \infty}\left(I_{1}^{2}+I_{2}^{2}\right) / T
$$

where $T$ is a long time and

$$
\begin{aligned}
& I_{1}=(1 / \pi) \int_{0}^{T} u \cos 2 \pi n t d t \\
& I_{2}=(1 / \pi) \int_{0}^{T} u \sin 2 \pi n t d t .
\end{aligned}
$$

When the fluctuations are superposed on a stream of mean velocity $U$ and are very small in comparison with $U$, the changes in $u$ at a fixed point may be regarded as due to the passage of a fixed turbulent pattern over the point, i.e., it may be assumed that

$$
u=\phi(t)=\phi(x / U)
$$

where $x$ is measured upstream at time $t=0$ from the fixed point. The correlation $R_{x}$ between the fluctuations at the times $t$ and $t+x / U$ is defined by

$$
R_{x}=\frac{\overline{\phi(t) \phi(t+x / U)}}{\overline{u^{2}}} .
$$

It can be shown ${ }^{20}$ that

$$
\int_{-\infty}^{\infty} \phi(t) \phi(t+x / U) d t=2 \pi^{2} \int_{0}^{\infty}\left(I_{1}^{2}+I_{2}^{2}\right) \cos (2 \pi n x / U) d n
$$

${ }^{20}$ Taylor, G. I., The spectrum of turbulence, Proc. Roy. Soc. London, Ser. A, 164, 476 (1938). 
or, substituting for $I_{1}^{2}+I_{2}^{2}$ its value in terms of $F(n)$,

$$
R_{x}=\int_{0}^{\infty} F(n) \cos (2 \pi n x / U) d n
$$

and

$$
F(n)=(4 / U) \int_{0}^{\infty} R_{x} \cos (2 \pi n x / U) d x
$$

In other words, the correlation coefficient $R_{x}$ and $U F(n) / \sqrt{8 \pi}$ are Fourier transforms. If either is measured, the other may be computed. $R_{x}$ is the function denoted by $f$ in section 13 . The length $\lambda$, which was defined in terms of the function $g$ or $R_{y}$, is related to $R_{x}$ by the equation:

$$
1 / \lambda^{2}=2 \lim _{x \rightarrow 0}\left(1-R_{x}\right) / x^{2} .
$$

When $n$ and $x$ are small, $\cos (2 \pi n x / U)$ in (19.6) may be approximated by $1-2 \pi^{2} x^{2} n^{2} / U^{2}$. Hence

$$
1 / \lambda^{2}=\left(4 \pi^{2} / U^{2}\right) \int_{0}^{\infty} n^{2} F(n) d n .
$$

If the turbulence is self-preserving, the shape of the correlation curve is a function of the Reynolds Number of the turbulence. Hence the spectrum curve is also a function of the Reynolds Number of the turbulence. Introducing the longitudinal scale $L_{x}\left(L_{x}=\int_{0}^{\infty} R_{x} d x\right)$ in equation (19.9),

$$
\frac{L_{x}^{2}}{\lambda^{2}}=4 \pi^{2} \int_{0}^{\infty}\left(\frac{n L_{x}}{U}\right)^{2} \frac{U F(n)}{L_{x}} d\left(\frac{n L_{x}}{U}\right)
$$

and in equation (19.7),

$$
\frac{U F(n)}{L_{x}}=4 \int_{0}^{\infty} R_{x} \cos \frac{2 \pi n L_{x}}{U} \frac{x}{L_{x}} d\left(\frac{x}{L_{x}}\right)
$$

both of which are expressed in terms of the non-dimensional variables $U F(x) / L_{x}, n L_{x} / U, x / L_{x}, \lambda / L_{x}$, and $R_{x}$. The mean speed $U$ enters only in fixing the frequency scale.

Typical spectrum curves determined experimentally ${ }^{21,22}$ are shown in Fig. 10. Studies of the relation between the spectrum and the correlation curve have been given by Taylor. ${ }^{20}$

From equation (19.10) it may be inferred that if the curve of $U F(n) / L_{x}$ vs.

${ }^{21}$ Simmons, L. F. G., and Salter, C., An experimental determination of the spectrum of turbulence, Proc. Roy. Soc. London Ser. A, 165, 73 (1938).

${ }^{22}$ Dryden, H. L., Turbulence investigations at the National Bureau of Standards, Proc. Fifth Inter. Congr. Appl. Mech., Cambridge, Mass., 1938, p. 362. 
$n L_{x} / U$ is independent of $U, L_{x} / \lambda$ should also be independent of $U$, which is contrary to the known dependence of $L_{x} / \lambda$ on the Reynolds Number of the turbulence. Equation (19.9) shows that the value of $\lambda$ is determined largely by the values of $F(n)$ at large values of $n$. The NPL measurements in Fig. 10
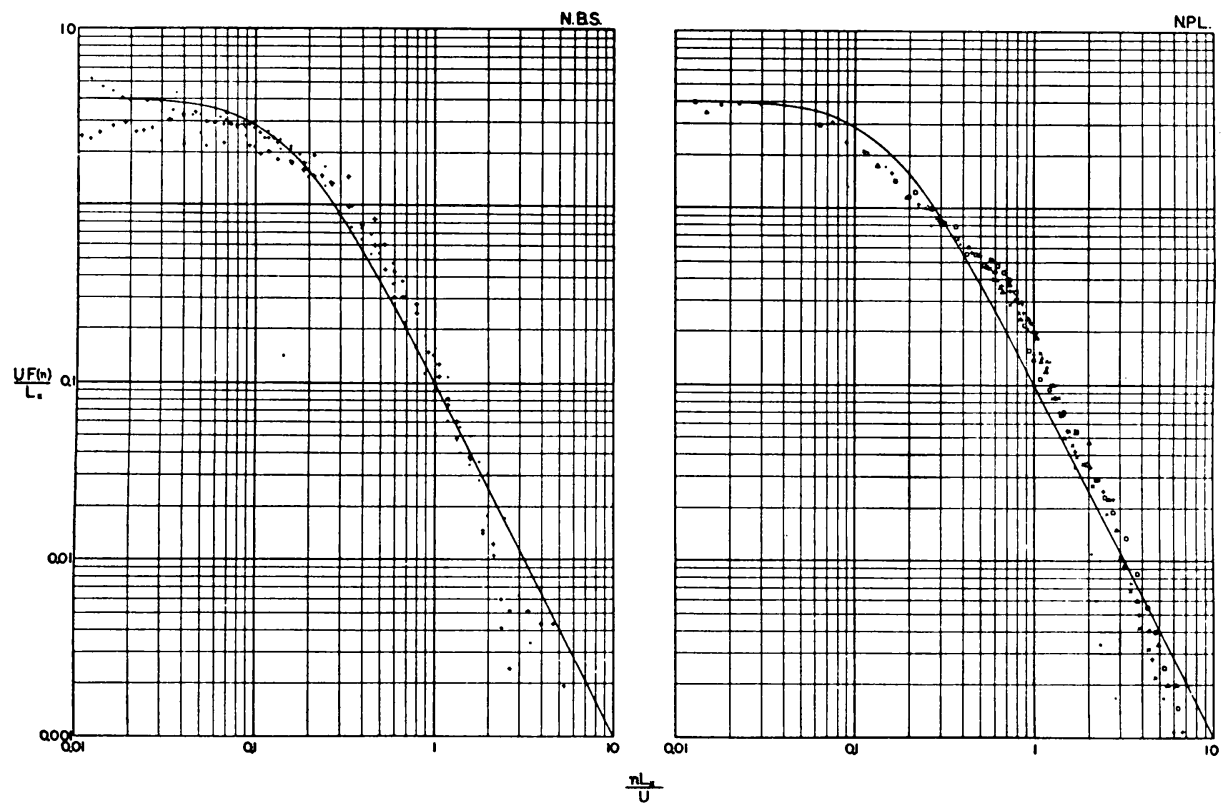

FIG. 10. Comparison of National Bureau of Standards and National Physical Laboratory measurements of the spectrum of turbulence, plotted non-dimensionally.

At left, NBS values $40(\cdot)$ and $160(+)$ inches behind 1 -inch mesh screen at $40 \mathrm{ft} / \mathrm{sec}$.

At right, NPL values of $\left(F(n)\right.$ from Table II of reference $21, L_{x}$ from reference 20) 82 inches behind 3-inch mesh screen at $15(\cdot), 20(X), 25(+), 30(\triangle)$, and $35(\square) \mathrm{ft} / \mathrm{sec}$.

The reference curve in each case is the curve

$$
\frac{U F(n)}{L_{x}}=\frac{4}{1+\frac{4 \pi^{2} n^{2} L_{x}^{2}}{U^{2}}}
$$

where $U$ is the mean speed, $L_{x}$ is the integral $\int_{0}^{\infty} R_{x} d x, R_{x}$ is the correlation between the fluctuations at two points separated by the distance $x$ in the direction of flow, $n$ is the frequency, and $F(n)$ is the fraction of the total energy of the turbulence arising from frequencies between $n$ and $n+d n$.

show clearly this dependence of the spectrum curve on $U$ at high frequencies.

When the Reynolds Number of the turbulence is large, $\lambda / L_{x}$ becomes small. Experimental measurements show that both $R_{x}$ and $R_{y}$ curves approach exponential curves. From integration of equation (13.9) it follows that $2 L=L_{x}$ and equation (19.11) for the corresponding spectrum curve becomes:

$$
\frac{U F(n)}{L_{x}}=\frac{4}{1+4 \pi^{2} n^{2} L_{x} / U^{2}} .
$$


This is the reference curve drawn in Fig. 10. As $U$ decreases, $\lambda$ increases, and the departures at large values of $n L_{x} / U$ becomes greater. The changes in the total energy of the fluctuations associated with these changes in the spectrum at high frequencies are extremely small.

Adopting this expression for the spectrum curve, it is possible to compute the effect of varying the cut-off frequency of the measuring equipment on the measured value of the energy of the fluctuations. If the equipment passes

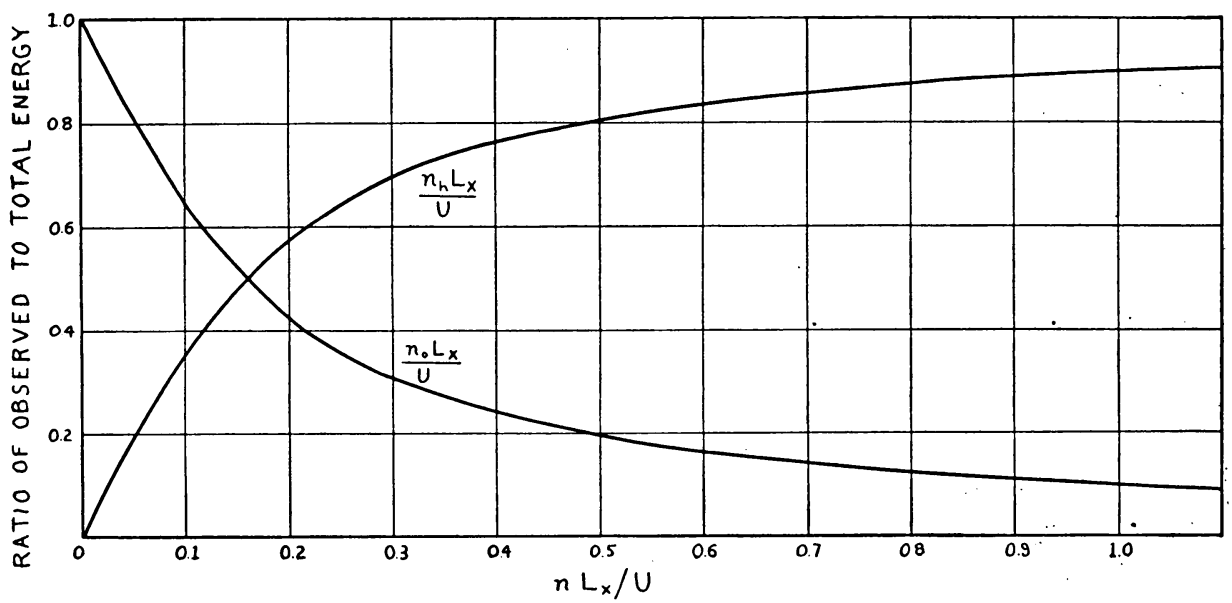

FIG. 11. Effect of cut-off frequencies of apparatus on observed energy of turbulence for spectrum given by reference curve of Fig. 9.

$n_{0}$ is the lower cut-off frequency, $n_{h}$ the upper cut-off frequency, $L_{x}$ the longitudinal scale, $U$ the mean speed.

high frequencies but cuts off sharply at a lower frequency $n_{0}$, the measured total energy is

$$
\frac{1}{2} \rho \overline{u^{2}} \int_{n_{0} L_{x} / U}^{\infty} \frac{4\left(L_{x} / U\right) d n}{1+4 \pi^{2} n^{2} L_{x}^{2} / U^{2}}=\left(1-\frac{4}{2 \pi} \tan ^{-1} \frac{2 \pi n_{0} L_{x}}{U}\right) \frac{1}{2} \rho \overline{u^{2}} .
$$

The ratio of the observed to the actual total energy is shown in Fig. 11 for various values of $n_{0} L_{x} / U$.

Similarly, if the equipment passes low frequencies but cuts off sharply at a higher frequency $n_{h}$, the measured total energy is $(4 / 2 \pi) \tan ^{-1} 2 \pi n_{h} L_{x} / U\left(\frac{1}{2} \rho \overline{u^{2}}\right)$. The ratio of the observed to the actual total energy for high frequency cutoff is also shown in Fig. 10.

The fact that the correlation and spectrum curves are of the exponential type has been interpreted ${ }^{22}$ as meaning that turbulence is a generalized chance phenomenon, as nearly chance as a continuous curve can be and retain its continuity. 
20. Fluctuating pressure gradients. In theories of the effect of turbulence on transition in boundary layers, it is desired to know the value of the rootmean-square pressure gradients, i.e., $\overline{(\partial p / \partial x)^{2}}, \overline{(\partial p / \partial y)^{2}}$, and $\overline{(\partial p / \partial z)^{2}}$. Taylor has shown ${ }^{8}$ that

$$
\sqrt{(\partial p / \partial x)^{2}}=2 \sqrt{2} \rho \overline{u^{2}} / \lambda .
$$

Combining this with the relation (18.11), i.e., $\lambda / L=6.97 / \sqrt{\left(\overline{u^{2}}\right)^{1 / 2} L / \nu}$

$$
\sqrt{\left(\frac{\partial p}{\partial x}\right)^{2}}=\frac{2 \sqrt{2} \rho \overline{u^{2}}}{6.97 L} \sqrt{\frac{(\bar{u})^{1 / 2} L}{\nu}} .
$$

The quantities $\sqrt{\overline{u^{2}}}$ and $L$ occur in this expression in the combination $\left[\left(\sqrt{\overline{u^{2}}}\right) / L^{1 / 5}\right]^{5 / 2}$. The ratio $\left(\sqrt{\overline{u^{2}}} / U\right)(D / L)^{1 / 5}$, where $U$ is the mean speed and $D$ the reference dimension of a body under study is known as the Taylor turbulence parameter.

21. The diffusive character of turbulence. An early experimental distinction between turbulent and non-turbulent flow was based on the observation that a filament of dye introduced into a turbulent fluid stream is rapidly diffused over the entire cross section of the stream whereas in a non-turbulent flow the filament retains its identity although it may show some waviness. It has been pointed out in section 7 that the effect of the turbulent fluctuations on the mean motion is the introduction of eddy stresses associated with the transfer of momentum by the diffusion of fluid particles. Von Kármán ${ }^{23}$ has given a useful account of the mechanism of the diffusion of discrete particles and its effect in producing a shearing stress. A theory of diffusion by continuous movements has been developed by Taylor. ${ }^{13}$ The process of diffusion has been found helpful in the experimental study of the statistical properties of turbulence.

22. Diffusion by continuous movements. Consider in a uniform isotropic turbulent field the displacement $X$ and velocity $u$ parallel to the arbitrarily selected $x$ axis. The intensity $\sqrt{\overline{u^{2}}}$ is constant, the field being assumed uniform. Let $u_{t}$ and $u_{t^{\prime}}$ be the values of $u$ at times $t$ and $t^{\prime}$ respectively. Consider the definite integral $\int_{0}^{t} u_{t} u_{t^{\prime}} d t^{\prime}$. Introducing the correlation coefficient $R_{t, t^{\prime}}$ between $u_{t}$ and $u_{t^{\prime}}$, remembering that $\overline{u^{2}}$ is constant,

$$
\int_{0}^{t} \overline{u_{t} u_{t^{\prime}}} d t^{\prime}=\overline{u^{2}} \int_{0}^{t} R_{t, t^{\prime}} d t^{\prime}
$$

Let $t^{\prime}-t=T$ and place $R_{t, t^{\prime}}=R_{T}$. Since $R_{T}$ is an even function of $T,(22.1)$ may be written:

$$
\int_{0}^{t} \overline{u_{t} u_{t^{\prime}}} d t^{\prime}=\overline{u^{2}} \int_{0}^{t} R_{T} d T .
$$

${ }^{23}$ Kármán, Th. von, Turbulence, Jour. Roy. Aeron. Soc. 41, 1109 (1937). 
But

$$
\int_{0}^{t} \overline{u_{t} u_{t^{\prime}}} d t^{\prime}=\overline{u_{t} \int_{0}^{t} u_{t^{\prime}} d t^{\prime}}=\overline{u_{t} X}=\overline{u X}
$$

Hence

$$
\overline{u^{2}} \int_{0}^{t} R_{T} d T=\overline{u X}=(1 / 2) d \overline{X^{2}} / d t .
$$

When the time $t$ is so small that $R_{T}$ approximates unity, equation (22.4) becomes:

or

$$
(1 / 2) d \overline{X^{2}} / d t=\overline{u^{2}} t
$$

$$
\sqrt{\overline{X^{2}}}=\sqrt{u^{2}} t .
$$

If $R_{T}$ is equal to zero for all times greater than some time $T_{0}$

$$
\overline{u X}=\overline{u^{2}} \int_{0}^{T_{0}} R_{T} d T=\text { constant }
$$

Define a length $l_{1}$ by the relation:

whence

$$
l_{1}=\sqrt{\overline{u^{2}}} \int_{0}^{T_{0}} R_{T} d T
$$

$$
l_{1} \sqrt{u^{2}}=\overline{u X}=(1 / 2) d \overline{X^{2}} / d t
$$

and

$$
\overline{X^{2}}=2 l_{1} \sqrt{u^{2}} t .
$$

If $R_{T}=e^{-T / T_{0}}, l_{1}=\sqrt{u^{2}} T_{0}$ and the solution of (22.4) yields:

$$
\overline{X^{2}}=2 \overline{u^{2}} T_{0}\left[t-T_{0}\left(1-e^{-T / T_{0}}\right)\right] .
$$

Equation (22.10) reduces to (22.5) when $t$ is small compared to $T_{0}$ and to (22.9) when $t$ is large compared to $T_{0}$.

The diffusion in a uniform field is accordingly completely determined by the correlation function $R_{T}$.

23. Diffusion in isotropic turbulence. The foregoing theory is directly applicable to diffusion in a uniform isotropic field. However no general statement can be made as to the relation between the length $l_{1}$ and the scale $L$ defined in terms of the correlation coefficient $R_{y}$ in section 9. For the turbulence behind a grid or honeycomb, Taylor found from an analysis of the available experimental results that $L$ was approximately twice $l_{1}$. 
The essential features of diffusion in isotropic turbulence expressed in equations (22.5) and (22.9) may be summarized as follows:

1. For time intervals which are small in comparison with the ratio of $l_{1}$ to $\sqrt{\overrightarrow{u^{2}}}$, the diffusing quantity spreads at a uniform rate proportional to the intensity $\sqrt{u^{2}}$, and the rate is not dependent on the length $l_{1}$.

2. For time intervals which are large in comparison with the ratio of $l_{1}$ to $\sqrt{u^{2}}$, the diffusing quantity $N$ spreads in accordance with the usual diffusion equation

$$
\frac{\partial N}{\partial t}+U \frac{\partial N}{\partial x}+V \frac{\partial N}{\partial y}+W \frac{\partial N}{\partial z}=\frac{\partial}{\partial x}\left(D \frac{\partial N}{\partial x}\right)+\frac{\partial}{\partial y}\left(D \frac{\partial N}{\partial y}\right)+\frac{\partial}{\partial z}\left(D \frac{\partial N}{\partial z}\right)
$$

with a coefficient of diffusion $D$ equal to $l_{1} \sqrt{\overline{u^{2}}}$, where $l_{1}$ is a length defined by $\int_{0}^{\infty} R_{T} d T$.

3. For intermediate time intervals, the diffusion is dependent on the function $R_{T}$ which represents the correlation between the speed of a particle at any instant and the speed of the same particle after a time interval $T$.

Consider the diffusion of heat from a hot wire placed in a uniform field of isotropic turbulence in a fluid stream of mean speed $U$. Observations of the lateral spread of the thermal wake at a distant $x$ downstream may be used to compute the root-mean-square lateral displacement $\sqrt{\overline{Y^{2}}}$ of the heated particles during a time interval $t=x / U$.

It is convenient to characterize the spread by the angle subtended at the source by the two positions where the temperature rise is half that at the center of the wake. There is a lateral spread of heat produced by the ordinary molecular conduction corresponding to an angle $\alpha_{0}$ in degrees of $190.8 \sqrt{k / \rho c U x}$ where $k, \rho$, and $c$ are thermal conductivity, density, and specific heat (at constant pressure) of the fluid. It may be shown that the total subtended angle $\alpha$ is related to the angle $\alpha_{t}$ produced by turbulent diffusion and $\alpha_{0}$, as follows:

$$
\alpha^{2}=\alpha_{t}^{2}+\alpha_{0}^{2} .
$$

The temperature distribution in the wake follows an "error" curve as does the amplitude of the turbulent velocity fluctuations, so that the lateral displacement $Y$ also has the same Gaussian frequency distribution. The value of the lateral spread at which the ordinate is half the maximum is $2.354 \sqrt{\overline{Y^{2}}}$ for this distribution. Hence, expressing $\alpha_{t}$ in degrees,

$$
\alpha_{t}=134.7 \sqrt{\overline{Y^{2}}} / x
$$

whence from (22.5) for small values of $x$,

$$
\alpha_{t}=134.7 \sqrt{\overline{v^{2}}} / U
$$

where $v$ is written in place of $u$ in (22.5) since the diffusion in the $v$ direction is being studied. 
Thus an experiment on thermal diffusion provides a method of measuring $\sqrt{v^{2}}$. The method was used by Schubauer ${ }^{24}$ who showed that $\alpha_{t}$ was independent of speed over the range 10 to $50 \mathrm{ft} / \mathrm{sec}$ and also independent of $x$ over the range $1 / 2$ to 6 inches.

From measurements at large values of $x$, it is theoretically possible to compute the correlation curve, $R_{T}$, vs. $T$. In any actual experiment, however, the intensity of the turbulence will decrease with $x$ to an extent that must be considered. As discussed by Taylor, ${ }^{8} R_{T}$ may then be considered a function of $\eta=\int_{0}^{T} \sqrt{v^{2}} d T=\int_{0}^{x}\left(\sqrt{v^{2}} / U\right) d x$. The equation analogous to (22.4) for $v$ and $Y$ becomes:

$$
(1 / 2)\left(U / \sqrt{\overline{v^{2}}}\right)\left(d \overline{Y^{2}} / d x\right)=\int_{0}^{\eta_{x}} R_{\eta} d \eta
$$

The correlation is given by the expression

$$
R_{\eta}=\frac{d}{d \eta}\left(\frac{U}{2 \sqrt{\bar{v}^{2}}} \frac{d \overline{Y^{2}}}{d x}\right)
$$

and thus involves a double differentiation of experimental curves, a process which is usually not very accurate.

24. Statistical theory of non-isotropic turbulence. In non-isotropic turbulence the description of the state of the turbulence becomes much more complex. The eddy shearing stresses do not vanish and the eddy normal stresses are not necessarily equal. Six quantities instead of one are required to specify the intensity. Similarly the correlation tensor cannot be expressed in terms of a single scalar function. In general six scalar functions are required. No theoretical investigation using these twelve functions has yet been carried out.

The exploration of this field is still in its earliest stages. Von Kármán 9,16 has given some discussion of energy transport and dissipation and vorticity transport, neglecting the triple correlations, and he has also presented a more detailed discussion of two-dimensional flow with constant shearing stress (Couette's problem). The advance of the theory is definitely handicapped by the absence of reliable experimental data on the twelve functions required to describe the state of turbulence.

25. Diffusion in non-isotropic turbulence. The only theoretical approach at present available for estimating the diffusion in non-isotropic turbulence is to consider the process as approximately equivalent to diffusion in isotropic turbulence of intensity equal to $\sqrt{\overline{v^{2}}}$ and scale $l^{\prime}$, where $v$ is the component in the direction in which the diffusion is studied and $l^{\prime}$ is the length defined by an equation analogous to (22.7), namely,

${ }^{24}$ Schubauer, G. B., A turbulence indicator utilizing the diffusion of heat, Tech. Rept. Nat. Adv. Comm. Aeron., No. 524 (1935). 


$$
l^{\prime}=\sqrt{v^{2}} \int_{0}^{\infty} R_{T} d t .
$$

In most experiments the length $l^{\prime}$ is not measured. Prandtl defined a mixing length $l$ in terms of the shearing stress $\tau$ by the relation:

$$
\tau=\rho l^{2}\left|\frac{d U}{d y}\right| \frac{d U}{d y} .
$$

This relation may be interpreted as an equation governing the diffusion of momentum with a coefficient of diffusion equal to $l^{2}|d U / d y|$. Prandtl in fact assumed $\sqrt{\overrightarrow{v^{2}}}$ proportional to $l|d U / d y|$ and incorporated the factors of proportionality in the length $l$. It is obvious that

$$
l^{2}|d U / d y|=l^{\prime} \sqrt{v^{2}} \text {. }
$$

The length $l$ can be obtained experimentally if the distributions of velocity and shearing stress are known, and, if $\sqrt{v^{2}}$ is also measured, $l^{\prime}$ may be computed. Sherwood and $W_{0 e r t z}{ }^{25}$ have made an experimental study of these relationships.

Taylor ${ }^{26}$ pointed out that fluctuating pressure gradients influence the transfer of momentum and suggested that the vorticity be taken as the property undergoing diffusion. The result was the well known vortex transport theory.

Both theories imply diffusion for a time interval long compared to $l^{\prime} \sqrt{v^{2}}$. When diffusion is studied near the source, experiment shows ${ }^{27}$ a behaviour like that discussed in section 23. The spread is nearly linear with $x$, although unsymmetical in this case. It is probable that the unsymmetrical character cannot be explained on the basis of a single scalar diffusion coefficient.

26. Correlation in turbulent flow through a pipe. Taylor ${ }^{28}$ has shown that the correlation between the component of velocity at a fixed point and that at a variable point in the same cross section must be negative for some positions of the variable point, if the applied pressure difference between the ends of the pipe is constant and the fluid may be considered incompressible. Suppose the mean velocity is $U$ and the correlation $R$ has been measured between

${ }^{25}$ Sherwood, T. K., and Woertz, B. B., Mass transfer between phases, role of eddy diffusion, Ind. Eng. Chem. 31, 1034 (1939).

${ }^{26}$ Taylor, G. I., Transport of vorticity and heat through fuids in turbulent motion, Proc. Roy. Soc. London Ser. A, 135, 685 (1932).

${ }^{27}$ Skramstad, H. K., and Schubauer, G. B., The application of thermal diffusion to the study of turbulent air flow, Phys. Rev., 53, 927 (1938). Abstract only. Full paper not published. A few additional details are given in Dryden, Hugh L., Turbulence and diffusion, Ind. Eng. Chem. 31, 416 (1939).

${ }^{28}$ Taylor, G. I., Correlation measurements in a turbulent flow through a pipe, Proc. Roy. Soc. London Ser. A, 157, 537 (1936). 
the component $u_{1}$ of the fluctuations at a fixed point $\mathrm{P}$ and $u_{2}$ at a variable point $Q$ in the same cross section. Since the mean flow is constant,

$$
\int\left(U+u_{2}\right) d y d z=\int U d y d z=\text { constant }
$$

where the integration is taken over the cross section. At any instant,

$$
\int u_{2} d y d z=0
$$

Multiplying by $u_{1}$, which is constant for this integration and may be placed under the integral sign,

$$
\int u_{1} u_{2} d y d z=0
$$

Since (26.3) is true for any instant, it is true for the integral over a time interval $T$. Hence

$$
(1 / T) \int_{0}^{T}\left[\int u_{1} u_{2} d y d z\right] d t=0 .
$$

Changing the order of the integration and remembering that $(1 / T) \int_{0}^{T} u_{1} u_{2} d t$ $=\overline{u_{1} u_{2}}$

$$
\int \overline{u_{1} u_{2}} d y d z=0
$$

Introducing the correlation $R$,

$$
\int R u_{1}^{\prime} u_{2}^{\prime} d y d z=0 .
$$

But $u_{1}^{\prime}$ is constant with respect to the integration and accordingly

$$
\int R u_{2}^{\prime} d y d z=0
$$

Since $u_{2}^{\prime}$ is positive, $R$ must be negative for some positions of $Q$.

For a circular pipe (26.7) becomes:

$$
\int_{0}^{a} u_{r}^{\prime} R r d r=0
$$

where $u_{r}^{\prime}$ is the value of $\sqrt{u^{2}}$ at radius $r$ and $a$ is the radius of the pipe.

This relation was experimentally verified in experiments made by Simmons with the fixed point at the center of the pipe. 